\title{
Rational Design of Nonbonded Point Charge Models for Highly Charged Metal Cations with Lennard-Jones 12-6 Potential
}

Yongguang Zhang ${ }^{\dagger}$ Yang Jiang , Yejie Qiu $\dagger^{\dagger}$, and Haiyang Zhang ${ }^{* \dagger}$

$\dagger$ Department of Biological Science and Engineering, School of Chemistry and Biological Engineering, University of Science and Technology Beijing, 100083 Beijing, China

$¥$ Department of Chemistry, Pennsylvania State University, University Park, Pennsylvania 16802,

United States

Corresponding Author

*zhanghy@ustb.edu.c 
Table S1. Simulated Real Hydration Free Energy (HFE, kcal/mol), Ion-Oxygen Distance (IOD, §̊), and Coordination Number (CN) of

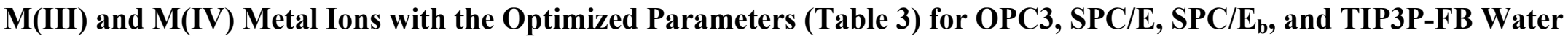

\begin{tabular}{|c|c|c|c|c|c|c|c|c|c|c|c|c|}
\hline & \multicolumn{3}{|c|}{ OPC3 } & \multicolumn{3}{|c|}{$\mathrm{SPC} / \mathrm{E}$} & \multicolumn{3}{|c|}{$\mathrm{SPC} / \mathrm{E}_{\mathrm{b}}$} & \multicolumn{3}{|c|}{ TIP3P-FB } \\
\hline & HFE & IOD & $\mathrm{CN}$ & HFE & IOD & $\mathrm{CN}$ & HFE & IOD & $\mathrm{CN}$ & HFE & IOD & $\mathrm{CN}$ \\
\hline $\mathrm{Al}^{3+}$ & $-1108.2 \pm 0.2$ & 1.89 & 6.0 & $-1108.1 \pm 0.1$ & 1.89 & 6.0 & $-1108.5 \pm 0.1$ & 1.89 & 6.0 & $-1108.0 \pm 0.2$ & 1.88 & 6.0 \\
\hline $\mathrm{Cr}^{3+}$ & $-985.1 \pm 0.2$ & 1.96 & 6.0 & $-984.9 \pm 0.2$ & 1.96 & 6.0 & $-983.7 \pm 0.1$ & 1.96 & 6.0 & $-984.5 \pm 0.1$ & 1.96 & 6.0 \\
\hline $\mathrm{Fe}^{3+}$ & $-1045.1 \pm 0.2$ & 2.03 & 6.7 & $-1045.4 \pm 0.2$ & 2.04 & 7.1 & $-1045.5 \pm 0.3$ & 2.04 & 7.1 & $-1046.1 \pm 0.4$ & 2.04 & 6.9 \\
\hline $\operatorname{In}^{3+}$ & $-976.4 \pm 0.4$ & 2.15 & 7.9 & $-977.6 \pm 0.4$ & 2.15 & 8.0 & $-978.6 \pm 0.4$ & 2.15 & 8.0 & $-977.3 \pm 0.5$ & 2.16 & 8.0 \\
\hline $\mathrm{Lu}^{3+}$ & $-866.1 \pm 0.3$ & 2.34 & 9.0 & $-866.5 \pm 0.2$ & 2.35 & 9.0 & $-866.1 \pm 0.1$ & 2.34 & 9.0 & $-866.2 \pm 0.1$ & 2.35 & 9.0 \\
\hline $\mathrm{Tl}^{3+}$ & $-974.2 \pm 0.3$ & 2.23 & 8.0 & $-975.0 \pm 0.3$ & 2.22 & 8.0 & $-974.7 \pm 0.2$ & 2.23 & 8.1 & $-975.0 \pm 0.1$ & 2.24 & 8.0 \\
\hline $\mathrm{Tm}^{3+}$ & $-866.3 \pm 0.3$ & 2.37 & 9.0 & $-866.4 \pm 0.2$ & 2.37 & 9.0 & $-865.8 \pm 0.3$ & 2.36 & 9.0 & $-866.4 \pm 0.2$ & 2.37 & 9.0 \\
\hline $\mathrm{Er}^{3+}$ & $-861.5 \pm 0.1$ & 2.37 & 9.0 & $-861.8 \pm 0.2$ & 2.37 & 9.0 & $-860.9 \pm 0.2$ & 2.36 & 9.0 & $-861.8 \pm 0.2$ & 2.37 & 9.0 \\
\hline $\mathrm{Y}^{3+}$ & $-850.0 \pm 0.1$ & 2.37 & 9.0 & $-851.0 \pm 0.2$ & 2.37 & 9.0 & $-850.7 \pm 0.5$ & 2.36 & 9.0 & $-851.0 \pm 0.4$ & 2.37 & 9.0 \\
\hline $\mathrm{Dy}^{3+}$ & $-844.6 \pm 0.1$ & 2.38 & 9.0 & $-844.4 \pm 0.1$ & 2.37 & 9.0 & $-844.3 \pm 0.2$ & 2.38 & 9.0 & $-844.6 \pm 0.2$ & 2.37 & 9.0 \\
\hline $\mathrm{Tb}^{3+}$ & $-838.8 \pm 0.2$ & 2.40 & 9.0 & $-838.0 \pm 0.2$ & 2.40 & 9.0 & $-838.3 \pm 0.2$ & 2.41 & 9.0 & $-838.4 \pm 0.2$ & 2.40 & 9.0 \\
\hline $\mathrm{Gd}^{3+}$ & $-831.9 \pm 0.2$ & 2.39 & 9.0 & $-831.9 \pm 0.3$ & 2.40 & 9.0 & $-833.2 \pm 0.2$ & 2.40 & 9.0 & $-832.8 \pm 0.1$ & 2.39 & 9.0 \\
\hline $\mathrm{Eu}^{3+}$ & $-830.1 \pm 0.2$ & 2.45 & 9.0 & $-828.3 \pm 0.2$ & 2.45 & 9.0 & $-828.8 \pm 0.2$ & 2.45 & 9.0 & $-828.7 \pm 0.2$ & 2.44 & 9.0 \\
\hline $\mathrm{Sm}^{3+}$ & $-820.4 \pm 0.2$ & 2.45 & 9.0 & $-819.7 \pm 0.2$ & 2.45 & 9.0 & $-820.6 \pm 0.2$ & 2.45 & 9.0 & $-820.2 \pm 0.3$ & 2.45 & 9.0 \\
\hline $\mathrm{Nd}^{3+}$ & $-809.9 \pm 0.3$ & 2.48 & 9.0 & $-809.2 \pm 0.1$ & 2.48 & 9.0 & $-810.0 \pm 0.2$ & 2.48 & 9.0 & $-810.1 \pm 0.3$ & 2.48 & 9.0 \\
\hline $\mathrm{Pr}^{3+}$ & $-800.3 \pm 0.2$ & 2.53 & 9.2 & $-802.3 \pm 0.2$ & 2.55 & 9.9 & $-801.2 \pm 0.3$ & 2.55 & 9.9 & $-802.2 \pm 0.2$ & 2.55 & 9.8 \\
\hline $\mathrm{Ce}^{3+}$ & $-789.8 \pm 0.1$ & 2.54 & 9.4 & $-790.7 \pm 0.1$ & 2.56 & 9.9 & $-790.1 \pm 0.2$ & 2.56 & 9.8 & $-790.4 \pm 0.2$ & 2.54 & 9.6 \\
\hline $\mathrm{La}^{3+}$ & $-777.5 \pm 0.1$ & 2.53 & 9.0 & $-777.6 \pm 0.3$ & 2.52 & 9.2 & $-777.6 \pm 0.2$ & 2.53 & 9.3 & $-777.3 \pm 0.2$ & 2.53 & 9.1 \\
\hline $\mathrm{Hf}^{4+}$ & $-1698.3 \pm 0.3$ & 2.16 & 8.0 & $-1697.1 \pm 0.3$ & 2.15 & 8.1 & $-1697.4 \pm 0.3$ & 2.15 & 8.0 & $-1698.3 \pm 0.3$ & 2.17 & 8.5 \\
\hline $\mathrm{Zr}^{4+}$ & $-1656.5 \pm 0.3$ & 2.20 & 8.8 & $-1655.5 \pm 0.2$ & 2.19 & 8.9 & $-1657.2 \pm 0.3$ & 2.2 & 9.0 & $-1656.4 \pm 0.2$ & 2.18 & 8.5 \\
\hline $\mathrm{Ce}^{4+}$ & $-1495.7 \pm 0.2$ & 2.42 & 10.0 & $-1496.8 \pm 0.3$ & 2.42 & 10.0 & $-1496.9 \pm 0.3$ & 2.42 & 10.0 & $-1496.3 \pm 0.4$ & 2.43 & 10.0 \\
\hline $\mathrm{Pu}^{4+}$ & $-1601.2 \pm 0.3$ & 2.39 & 10.0 & $-1600.9 \pm 0.3$ & 2.38 & 10.0 & $-1601.1 \pm 0.6$ & 2.38 & 10.0 & $-1601.1 \pm 0.3$ & 2.38 & 10.0 \\
\hline $\mathrm{U}^{4+}$ & $-1553.5 \pm 0.5$ & 2.43 & 10.0 & $-1554.1 \pm 0.5$ & 2.42 & 10.0 & $-1553.1 \pm 0.2$ & 2.43 & 10.0 & $-1553.3 \pm 0.4$ & 2.42 & 10.0 \\
\hline $\mathrm{Th}^{4+}$ & $-1424.0 \pm 0.4$ & 2.52 & 10.4 & $-1424.5 \pm 0.3$ & 2.53 & 11.1 & $-1422.6 \pm 0.3$ & 2.54 & 11.1 & $-1423.4 \pm 0.6$ & 2.52 & 10.9 \\
\hline RMSE & 0.6 & 0.01 & & 0.6 & 0.01 & & 0.5 & 0.01 & & 0.3 & 0.01 & \\
\hline MAE & 0.4 & 0.00 & & 0.5 & 0.01 & & 0.4 & 0.01 & & 0.3 & 0.01 & \\
\hline
\end{tabular}


Table S2. Simulated Real Hydration Free Energy (HFE, kcal/mol), Ion-Oxygen Distance (IOD, Å), and Coordination Number (CN) of M(III) and M(IV) Metal Ions with the Optimized Parameters (Table 5) for OPC, TIP4P/2005, TIP4P-D, and TIP4P-FB Water

\begin{tabular}{|c|c|c|c|c|c|c|c|c|c|c|c|c|}
\hline \multirow[b]{3}{*}{$\mathrm{Al}^{3+}$} & \multicolumn{3}{|c|}{$\mathrm{OPC}$} & \multicolumn{3}{|c|}{ TIP4P/2005 } & \multicolumn{3}{|c|}{ TIP4P-D } & \multicolumn{3}{|c|}{ TIP4P-FB } \\
\hline & HFE & IOD & $\mathrm{CN}$ & HFE & IOD & $\mathrm{CN}$ & HFE & IOD & $\mathrm{CN}$ & HFE & IOD & $\mathrm{CN}$ \\
\hline & $-1107.5 \pm 0.2$ & 1.88 & 6.0 & $-1107.4 \pm 0.2$ & 1.89 & 6.0 & $-1107.2 \pm 0.2$ & 1.88 & 6.0 & $-1107.7 \pm 0.2$ & 1.89 & 6.0 \\
\hline $\mathrm{Cr}^{3+}$ & $-984.3 \pm 0.1$ & 1.97 & 6.0 & $-985.2 \pm 0.2$ & 1.96 & 6.0 & $-985.1 \pm 0.2$ & 1.96 & 6.0 & $-984.8 \pm 0.2$ & 1.96 & 6.0 \\
\hline $\mathrm{Fe}^{3+}$ & $-1044.1 \pm 0.2$ & 2.02 & 6.6 & $-1046.0 \pm 0.3$ & 2.04 & 7.0 & $-1044.6 \pm 0.5$ & 2.03 & 6.7 & $-1044.9 \pm 0.6$ & 2.04 & 6.7 \\
\hline $\operatorname{In}^{3+}$ & $-976.3 \pm 0.2$ & 2.17 & 7.9 & $-978.1 \pm 0.3$ & 2.16 & 8.0 & $-976.5 \pm 0.4$ & 2.17 & 7.8 & $-976.7 \pm 0.4$ & 2.17 & 8.0 \\
\hline $\mathrm{Lu}^{3+}$ & $-865.4 \pm 0.2$ & 2.34 & 9.0 & $-865.4 \pm 0.2$ & 2.34 & 9.0 & $-865.8 \pm 0.1$ & 2.34 & 8.4 & $-865.1 \pm 0.2$ & 2.34 & 9.0 \\
\hline $\mathrm{Tl}^{3+}$ & $-975.8 \pm 0.1$ & 2.24 & 8.0 & $-974.6 \pm 0.3$ & 2.23 & 8.0 & $-975.3 \pm 0.2$ & 2.23 & 8.0 & $-974.1 \pm 0.2$ & 2.23 & 8.0 \\
\hline $\mathrm{Tm}^{3+}$ & $-865.5 \pm 0.1$ & 2.37 & 9.0 & $-865.9 \pm 0.1$ & 2.37 & 9.0 & $-866.6 \pm 0.2$ & 2.36 & 9.0 & $-865.2 \pm 0.3$ & 2.36 & 9.0 \\
\hline $\mathrm{Er}^{3+}$ & $-860.5 \pm 0.3$ & 2.37 & 9.0 & $-861.2 \pm 0.3$ & 2.37 & 9.0 & $-861.6 \pm 0.1$ & 2.37 & 9.0 & $-860.8 \pm 0.3$ & 2.36 & 9.0 \\
\hline $\mathrm{Y}^{3+}$ & $-849.7 \pm 0.2$ & 2.37 & 9.0 & $-851.3 \pm 0.2$ & 2.36 & 9.0 & $-851.2 \pm 0.1$ & 2.37 & 9.0 & $-849.9 \pm 0.1$ & 2.37 & 9.0 \\
\hline $\mathrm{Dy}^{3+}$ & $-844.3 \pm 0.1$ & 2.38 & 9.0 & $-844.5 \pm 0.2$ & 2.37 & 9.0 & $-845.6 \pm 0.2$ & 2.38 & 9.0 & $-844.0 \pm 0.2$ & 2.37 & 9.0 \\
\hline $\mathrm{Tb}^{3+}$ & $-838.5 \pm 0.2$ & 2.41 & 9.0 & $-838.5 \pm 0.2$ & 2.40 & 9.0 & $-839.4 \pm 0.3$ & 2.41 & 9.0 & $-837.9 \pm 0.3$ & 2.41 & 9.0 \\
\hline $\mathrm{Gd}^{3+}$ & $-833.8 \pm 0.1$ & 2.40 & 9.0 & $-833.0 \pm 0.2$ & 2.40 & 9.0 & $-833.0 \pm 0.3$ & 2.39 & 9.0 & $-833.0 \pm 0.2$ & 2.39 & 9.0 \\
\hline $\mathrm{Eu}^{3+}$ & $-829.6 \pm 0.2$ & 2.45 & 9.0 & $-829.0 \pm 0.2$ & 2.44 & 9.0 & $-828.5 \pm 0.2$ & 2.45 & 9.0 & $-829.2 \pm 0.2$ & 2.46 & 9.0 \\
\hline $\mathrm{Sm}^{3+}$ & $-820.6 \pm 0.2$ & 2.45 & 9.0 & $-820.5 \pm 0.1$ & 2.44 & 9.0 & $-820.5 \pm 0.1$ & 2.45 & 9.0 & $-821.5 \pm 0.3$ & 2.46 & 9.0 \\
\hline $\mathrm{Nd}^{3+}$ & $-809.6 \pm 0.2$ & 2.47 & 9.0 & $-810.1 \pm 0.1$ & 2.47 & 9.0 & $-810.5 \pm 0.2$ & 2.48 & 9.0 & $-810.1 \pm 0.2$ & 2.47 & 9.0 \\
\hline $\operatorname{Pr}^{3+}$ & $-800.6 \pm 0.1$ & 2.54 & 9.2 & $-802.7 \pm 0.2$ & 2.55 & 9.9 & $-801.9 \pm 0.1$ & 2.55 & 9.3 & $-801.1 \pm 0.2$ & 2.55 & 9.8 \\
\hline $\mathrm{Ce}^{3+}$ & $-789.9 \pm 0.1$ & 2.54 & 9.2 & $-790.8 \pm 0.1$ & 2.56 & 10.0 & $-790.0 \pm 0.2$ & 2.55 & 9.2 & $-790.2 \pm 0.2$ & 2.56 & 9.8 \\
\hline $\mathrm{La}^{3+}$ & $-776.7 \pm 0.1$ & 2.53 & 9.0 & $-777.6 \pm 0.2$ & 2.54 & 9.7 & $-778.0 \pm 0.1$ & 2.52 & 9.0 & $-777.8 \pm 0.1$ & 2.53 & 9.2 \\
\hline $\mathrm{Hf}^{4+}$ & $-1698.1 \pm 0.3$ & 2.15 & 8.0 & $-1699.0 \pm 0.3$ & 2.15 & 8.0 & $-1697.4 \pm 0.4$ & 2.16 & 8.0 & $-1698.0 \pm 0.2$ & 2.15 & 8.0 \\
\hline $\mathrm{Zr}^{4+}$ & $-1656.3 \pm 0.2$ & 2.19 & 8.0 & $-1656.8 \pm 0.2$ & 2.18 & 8.2 & $-1657.0 \pm 0.3$ & 2.19 & 8.0 & $-1655.7 \pm 0.3$ & 2.18 & 8.2 \\
\hline $\mathrm{Ce}^{4+}$ & $-1496.0 \pm 0.1$ & 2.43 & 10.0 & $-1495.8 \pm 0.2$ & 2.43 & 10.0 & $-1496.5 \pm 0.2$ & 2.42 & 9.8 & $-1495.3 \pm 0.3$ & 2.43 & 10.0 \\
\hline $\mathrm{Pu}^{4+}$ & $-1600.5 \pm 0.3$ & 2.40 & 10.0 & $-1601.8 \pm 0.4$ & 2.40 & 10.0 & $-1601.7 \pm 0.3$ & 2.4 & 9.9 & $-1602.3 \pm 0.4$ & 2.39 & 10.0 \\
\hline $\mathrm{U}^{4+}$ & $-1552.5 \pm 0.3$ & 2.42 & 10.0 & $-1553.1 \pm 0.4$ & 2.41 & 10.0 & $-1553.4 \pm 0.3$ & 2.42 & 10.0 & $-1554.0 \pm 0.4$ & 2.41 & 10.0 \\
\hline $\mathrm{Th}^{4+}$ & $-1422.8 \pm 0.2$ & 2.52 & 10.6 & $-1423.8 \pm 0.2$ & 2.54 & 11.1 & $-1422.5 \pm 0.2$ & 2.52 & 10.0 & $-1424.2 \pm 0.3$ & 2.52 & 10.9 \\
\hline
\end{tabular}


Table S3. Comparison of van der Waals Parameters (R) of M(III) and M(IV) Metal Models with the Shannon's Revised Ion Effective Radii $^{a}$

\begin{tabular}{|c|c|c|c|c|c|c|c|c|c|c|c|c|c|}
\hline \multirow[t]{2}{*}{ water model } & \multicolumn{4}{|c|}{$\mathrm{M}(\mathrm{III})$} & \multicolumn{4}{|c|}{$\mathrm{M}(\mathrm{IV})$} & \multicolumn{4}{|c|}{$\mathrm{M}(\mathrm{III})+\mathrm{M}(\mathrm{IV})$} & \multirow[t]{2}{*}{ note $^{c}$} \\
\hline & RMSE & MSE & $\mathrm{PCC}^{b}$ & $\mathrm{SRCC}^{b}$ & RMSE & MSE & $\mathrm{PCC}^{b}$ & $\mathrm{SRCC}^{b}$ & RMSE & MSE & $\mathrm{PCC}^{b}$ & $\mathrm{SRCC}^{b}$ & \\
\hline OPC3 & 0.47 & 0.47 & 0.98 & 1.00 & 0.37 & 0.37 & 0.90 & 0.90 & 0.45 & 0.44 & 0.93 & 0.94 & HFE set \\
\hline $\mathrm{SPC} / \mathrm{E}$ & 0.46 & 0.46 & 0.98 & 1.00 & 0.35 & 0.35 & 0.89 & 0.90 & 0.44 & 0.43 & 0.93 & 0.94 & HFE set \\
\hline TIP3P & 0.46 & 0.46 & 0.98 & 1.00 & 0.34 & 0.34 & 0.89 & 0.90 & 0.43 & 0.43 & 0.92 & 0.94 & HFE set \\
\hline TIP3P-FB & 0.45 & 0.45 & 0.98 & 1.00 & 0.35 & 0.35 & 0.90 & 0.90 & 0.43 & 0.42 & 0.93 & 0.94 & HFE set \\
\hline $\mathrm{OPC}$ & 0.40 & 0.40 & 0.98 & 1.00 & 0.29 & 0.28 & 0.89 & 0.90 & 0.38 & 0.37 & 0.92 & 0.91 & HFE set \\
\hline TIP4P-Ew & 0.37 & 0.37 & 0.98 & 1.00 & 0.24 & 0.23 & 0.89 & 0.90 & 0.35 & 0.34 & 0.92 & 0.91 & HFE set \\
\hline TIP4P-FB & 0.40 & 0.39 & 0.98 & 1.00 & 0.28 & 0.28 & 0.89 & 0.90 & 0.37 & 0.36 & 0.92 & 0.92 & HFE set \\
\hline OPC3 & 0.64 & 0.64 & 0.98 & 0.98 & 0.72 & 0.72 & 0.96 & 1.00 & 0.67 & 0.66 & 0.95 & 0.91 & IOD set \\
\hline SPC/E & 0.64 & 0.64 & 0.99 & 0.98 & 0.72 & 0.71 & 0.97 & 1.00 & 0.66 & 0.65 & 0.96 & 0.92 & IOD set \\
\hline TIP3P & 0.64 & 0.64 & 0.99 & 0.98 & 0.71 & 0.71 & 0.97 & 1.00 & 0.66 & 0.65 & 0.96 & 0.92 & IOD set \\
\hline TIP3P-FB & 0.64 & 0.64 & 0.98 & 0.98 & 0.72 & 0.72 & 0.96 & 1.00 & 0.66 & 0.66 & 0.95 & 0.91 & IOD set \\
\hline $\mathrm{OPC}$ & 0.62 & 0.61 & 0.98 & 0.98 & 0.70 & 0.70 & 0.96 & 1.00 & 0.64 & 0.63 & 0.95 & 0.91 & IOD set \\
\hline TIP4P-Ew & 0.63 & 0.62 & 0.99 & 0.98 & 0.69 & 0.69 & 0.97 & 1.00 & 0.65 & 0.64 & 0.96 & 0.94 & IOD set \\
\hline TIP4P-FB & 0.63 & 0.62 & 0.98 & 0.98 & 0.71 & 0.70 & 0.96 & 1.00 & 0.65 & 0.64 & 0.95 & 0.91 & IOD set \\
\hline OPC3 & 0.68 & 0.67 & 0.98 & 0.97 & 0.79 & 0.78 & 0.92 & 0.74 & 0.71 & 0.70 & 0.90 & 0.84 & $12-6-4$ set \\
\hline $\mathrm{SPC} / \mathrm{E}$ & 0.69 & 0.68 & 0.98 & 0.98 & 0.80 & 0.80 & 0.94 & 0.90 & 0.71 & 0.71 & 0.89 & 0.87 & $12-6-4$ set \\
\hline TIP3P & 0.69 & 0.68 & 0.98 & 0.97 & 0.80 & 0.80 & 0.94 & 0.90 & 0.72 & 0.71 & 0.90 & 0.86 & $12-6-4$ set \\
\hline TIP3P-FB & 0.68 & 0.68 & 0.98 & 0.97 & 0.79 & 0.79 & 0.94 & 0.90 & 0.71 & 0.71 & 0.90 & 0.87 & $12-6-4$ set \\
\hline $\mathrm{OPC}$ & 0.66 & 0.66 & 0.97 & 0.97 & 0.78 & 0.77 & 0.93 & 0.90 & 0.69 & 0.69 & 0.89 & 0.85 & $12-6-4$ set \\
\hline TIP4P-Ew & 0.68 & 0.68 & 0.98 & 0.98 & 0.80 & 0.80 & 0.92 & 0.81 & 0.71 & 0.71 & 0.89 & 0.85 & $12-6-4$ set \\
\hline
\end{tabular}




\begin{tabular}{|c|c|c|c|c|c|c|c|c|c|c|c|c|c|}
\hline TIP4P-FB & 0.68 & 0.67 & 0.98 & 0.97 & 0.79 & 0.78 & 0.93 & 0.90 & 0.70 & 0.70 & 0.89 & 0.87 & $12-6-4$ set \\
\hline a99SB-disp & 0.22 & -0.20 & 0.99 & 1.00 & 0.26 & -0.26 & 0.99 & 0.99 & 0.23 & -0.21 & 0.98 & 0.96 & this work \\
\hline OPC3 & 0.14 & -0.05 & 0.98 & 0.99 & 0.21 & -0.20 & 0.99 & 0.99 & 0.16 & -0.09 & 0.96 & 0.93 & this work \\
\hline SPC/E & 0.15 & -0.10 & 0.98 & 1.00 & 0.22 & -0.22 & 0.99 & 0.99 & 0.17 & -0.13 & 0.97 & 0.94 & this work \\
\hline $\begin{array}{l}\mathrm{SPC} / \mathrm{E}_{\mathrm{b}} \\
\text { continued (Ta }\end{array}$ & $\begin{array}{l}0.15 \\
\text { le S3) }\end{array}$ & -0.10 & 0.98 & 1.00 & 0.22 & -0.21 & 0.99 & 0.99 & 0.17 & -0.13 & 0.97 & 0.95 & this work \\
\hline \multirow{2}{*}{ water model } & \multicolumn{4}{|c|}{$\mathrm{M}(\mathrm{III})$} & \multicolumn{4}{|c|}{$\mathrm{M}(\mathrm{IV})$} & \multicolumn{4}{|c|}{$\mathrm{M}(\mathrm{III})+\mathrm{M}(\mathrm{IV})$} & note $^{c}$ \\
\hline & RMSE & MSE & $\mathrm{PCC}^{b}$ & $\mathrm{SRCC}^{b}$ & RMSE & MSE & $\mathrm{PCC}^{b}$ & $\mathrm{SRCC}^{b}$ & RMSE & MSE & $\mathrm{PCC}^{b}$ & $\mathrm{SRCC}^{b}$ & \\
\hline TIP3P & 0.16 & -0.12 & 0.98 & 1.00 & 0.23 & -0.23 & 1.00 & 0.99 & 0.18 & -0.14 & 0.97 & 0.94 & this work \\
\hline TIP3P-FB & 0.15 & -0.11 & 0.98 & 1.00 & 0.22 & -0.22 & 0.99 & 0.99 & 0.17 & -0.14 & 0.97 & 0.94 & this work \\
\hline $\mathrm{OPC}$ & 0.17 & -0.14 & 0.99 & 1.00 & 0.24 & -0.24 & 0.99 & 0.99 & 0.19 & -0.17 & 0.98 & 0.95 & this work \\
\hline TIP4P-D & 0.22 & -0.20 & 0.99 & 1.00 & 0.26 & -0.26 & 0.99 & 0.99 & 0.23 & -0.22 & 0.98 & 0.96 & this work \\
\hline TIP4P-Ew & 0.21 & -0.20 & 0.99 & 1.00 & 0.26 & -0.26 & 0.99 & 0.99 & 0.22 & -0.21 & 0.98 & 0.96 & this work \\
\hline TIP4P-FB & 0.19 & -0.17 & 0.99 & 1.00 & 0.24 & -0.24 & 0.99 & 0.99 & 0.20 & -0.18 & 0.98 & 0.95 & this work \\
\hline TIP4P/2005 & 0.22 & -0.22 & 0.99 & 1.00 & 0.26 & -0.26 & 0.99 & 0.99 & 0.23 & -0.23 & 0.98 & 0.96 & this work \\
\hline
\end{tabular}

${ }^{a}$ The Shannon's data were taken from Ref; ${ }^{1}$ Root-mean-square error (RMSE) and mean signed error (MSE) are in units of $\AA$; ${ }^{b}$ PCC stands for Pearson correlation coefficient and SRCC for Spearman rank correlation coefficient; ${ }^{c} R$ parameters of the 12-6 HFE, 12-6 IOD, and 12-6-4 sets were taken from Li and Merz..$^{2,3}$ 
Table S4. Lennard-Jones Parameters of the Oxygen Atom in the Water Models Used in This Work

\begin{tabular}{|c|c|c|c|c|c|c|c|}
\hline \multirow[t]{2}{*}{ model } & & $\mathrm{C} 6$ & $\mathrm{C} 12$ & $\sigma$ & $\varepsilon$ & $R$ & $\varepsilon$ \\
\hline & & $\left(10^{-3} \mathrm{~kJ} \mathrm{~mol}^{-1} \mathrm{~nm}^{6}\right)$ & $\left(10^{-6} \mathrm{~kJ} \mathrm{~mol}^{-1} \mathrm{~nm}^{12}\right)$ & $(\mathrm{nm})$ & $(\mathrm{kJ} / \mathrm{mol})$ & $(\AA)$ & $(\mathrm{kcal} / \mathrm{mol})$ \\
\hline \multirow[t]{5}{*}{ 3-site } & OPC3 & 2.79758 & 2.86185 & 0.31743 & 0.68369 & 1.781499 & 0.163406 \\
\hline & $\mathrm{SPC} / \mathrm{E}$ & 2.61882 & 2.63522 & 0.31656 & 0.65063 & 1.7767 & 0.1553 \\
\hline & $\mathrm{SPC} / \mathrm{E}_{\mathrm{b}}$ & 2.61882 & 2.63522 & 0.31656 & 0.65063 & 1.7767 & 0.1553 \\
\hline & TIP3P & 2.48971 & 2.43510 & 0.31506 & 0.63639 & 1.7683 & 0.152 \\
\hline & TIP3P-FB & 2.68716 & 2.76811 & 0.31780 & 0.65214 & 1.7835723 & 0.155866 \\
\hline \multirow[t]{6}{*}{ 4-site } & a99SB-disp & 4.01664 & 4.03743 & 0.31650 & 0.99899 & 1.7763 & 0.238764 \\
\hline & $\mathrm{OPC}$ & 3.59040 & 3.61961 & 0.31666 & 0.89036 & 1.777167268 & 0.212800813 \\
\hline & TIP4P-D & 2.55190 & 2.51041 & 0.31537 & 0.64852 & 1.7763 & 0.223841 \\
\hline & TIP4P/2005 & 3.07965 & 3.05997 & 0.31589 & 0.77487 & 1.77287 & 0.185197 \\
\hline & TIP4P-Ew & 2.73451 & 2.74528 & 0.31644 & 0.68095 & 1.7699 & 0.155 \\
\hline & TIP4P-FB & 3.01577 & 3.03455 & 0.31656 & 0.74928 & 1.77660486 & 0.179082 \\
\hline \multirow{2}{*}{\multicolumn{3}{|c|}{$\begin{array}{l}\text { exp. } \\
{ }^{a} \text { Taken from ref. }{ }^{4} b \text { From ref. }{ }^{5}\end{array}$}} & & & \multirow{2}{*}{\multicolumn{3}{|c|}{$1.4^{b}$}} \\
\hline & & & & & & & \\
\hline
\end{tabular}


Table S5. Water Properties from Experiment and Calculated from MD Simulations Using Different Methods for vdW Interactions

\begin{tabular}{llcccccc}
\hline water model & vdw-type $^{a}$ & $d_{\mathrm{OO}}(\AA)^{b}$ & density $(\mathrm{g} / \mathrm{L})$ & $E_{\mathrm{i}}(\mathrm{kcal} / \mathrm{mol})^{c}$ & $D_{\mathrm{PBC}}\left(\mathrm{x} 10^{-5} \mathrm{~cm}^{2} / \mathrm{s}\right)^{d}$ & $D_{0}\left(\mathrm{x} 10^{-5} \mathrm{~cm}^{2} / \mathrm{s}\right)^{d}$ & $v^{2} \mathrm{vcosity}(\mathrm{mPa} \cdot \mathrm{s})^{e}$ \\
TIP3P & cut-off & 2.78 & $985.9 \pm 0.6$ & $-9.59 \pm 0.01$ & $5.49 \pm 0.13$ & $6.13 \pm 0.13$ & $0.31 \pm 0.01$ \\
TIP3P & LJ-PME & 2.77 & $987.1 \pm 0.5$ & $-9.59 \pm 0.01$ & $5.43 \pm 0.14$ & $6.07 \pm 0.15$ & $0.31 \pm 0.01$ \\
TIP4P/2005 & cut-off & 2.77 & $996.7 \pm 0.7$ & $-11.44 \pm 0.01$ & $2.07 \pm 0.05$ & $2.31 \pm 0.05$ & $0.83 \pm 0.01$ \\
TIP4P/2005 & LJ-PME & 2.77 & $996.6 \pm 1.0$ & $-11.43 \pm 0.01$ & $2.09 \pm 0.06$ & $2.34 \pm 0.06$ & $0.82 \pm 0.01$ \\
OPC & cut-off & 2.81 & $997.5 \pm 0.2$ & $-12.31 \pm 0.01$ & $2.14 \pm 0.08$ & $2.39 \pm 0.08$ & $0.81 \pm 0.01$ \\
OPC & LJ-PME & 2.80 & $997.5 \pm 0.4$ & $-12.31 \pm 0.01$ & $2.14 \pm 0.08$ & $2.40 \pm 0.08$ & $0.81 \pm 0.02$ \\
exp & & $2.80^{f}$ & $996^{g}$ & $-9.92^{h}$ & & $2.299^{i}$ & $0.89 j$
\end{tabular}

${ }^{a}$ The type of treating vdW interactions by the cut-off scheme with a dispersion correction of energy and pressure and by the Lennard-Jones PME (LJ-PME) scheme. ${ }^{6,7 b}$ Oxygen-oxygen distance of water molecules. ${ }^{C}$ Interaction energy of water molecules. ${ }^{c}$ Diffusion constants from PBC MD simulations $\left(D_{\mathrm{PBC}}\right)$ and at infinite aqueous solution $\left(D_{0}\right)$. The simulated systems contained 1000 water molecules in a cubic box with a length of $\sim 31.1 \AA$. $D_{\mathrm{PBC}}$ was corrected to obtain $D_{0}$ using the box length and water viscosity (eq 5). ${ }^{e}$ Computed from non-equilibrium simulations with an external force ${ }^{8,9}$ using the GROMACS 5.1 .4 software. ${ }^{f}$ Taken from ref. ${ }^{10}{ }^{g}$ From ref. ${ }^{11 ~ h}$ From ref. ${ }^{12}{ }^{i}$ From ref. ${ }^{13} j$ From ref. ${ }^{14,15}$ 
Table S6. Calculated Osmotic Pressure (bar) and Osmotic Coefficient $(\varphi)$ of Trivalent Chlorides in Aqueous Solution Using TIP3PCompatible Ion Models ${ }^{a}$

\begin{tabular}{|c|c|c|c|c|c|c|c|c|c|}
\hline \multirow[t]{2}{*}{ molarity } & \multicolumn{3}{|c|}{$\mathrm{AlCl}_{3}$} & \multicolumn{3}{|c|}{$\mathrm{CeCl}_{3}$} & \multicolumn{3}{|c|}{$\mathrm{LaCl}_{3}$} \\
\hline & osmotic pressure & calculated $\varphi$ & $\varphi_{\exp }$ & osmotic pressure & calculated $\varphi$ & $\varphi_{\exp }$ & osmotic pressure & calculated $\varphi$ & $\varphi_{\exp }$ \\
\hline 0.2 & $13.4 \pm 1.0$ & $0.69 \pm 0.05$ & 0.85 & $16.1 \pm 1.4$ & $0.85 \pm 0.07$ & 0.80 & $17.9 \pm 1.1$ & $0.94 \pm 0.06$ & 0.80 \\
\hline 0.5 & $24.3 \pm 2.5$ & $0.52 \pm 0.05$ & 0.98 & $43.7 \pm 1.8$ & $0.97 \pm 0.04$ & 0.90 & $45.2 \pm 1.9$ & $1.00 \pm 0.04$ & 0.89 \\
\hline 1.0 & $37.4 \pm 2.1$ & $0.41 \pm 0.02$ & 1.30 & $96.3 \pm 3.0$ & $1.15 \pm 0.04$ & 1.08 & $92.3 \pm 3.3$ & $1.10 \pm 0.04$ & 1.07 \\
\hline 1.5 & $46.8 \pm 1.8$ & $0.36 \pm 0.01$ & 1.67 & $158.3 \pm 4.1$ & $1.33 \pm 0.03$ & 1.26 & $146.8 \pm 3.8$ & $1.23 \pm 0.03$ & 1.27 \\
\hline 2.0 & $43.5 \pm 2.4$ & $0.26 \pm 0.01$ & 2.08 & $222.9 \pm 6.9$ & $1.48 \pm 0.05$ & 1.46 & $221.4 \pm 6.9$ & $1.46 \pm 0.05$ & 1.46 \\
\hline 2.5 & $47.5 \pm 3.0$ & $0.23 \pm 0.02$ & 2.55 & $321.1 \pm 6.7$ & $1.77 \pm 0.04$ & 1.67 & $284.6 \pm 7.5$ & $1.56 \pm 0.04$ & 1.66 \\
\hline \multirow[t]{3}{*}{3.0} & $52.9 \pm 2.6$ & $0.22 \pm 0.01$ & 3.02 & & & & & & \\
\hline & \multicolumn{3}{|c|}{$\mathrm{NdCl}_{3}$} & \multicolumn{3}{|c|}{$\mathrm{PrCl}_{3}$} & \multicolumn{3}{|c|}{$\mathrm{YCl}_{3}$} \\
\hline & osmotic pressure & calculated $\varphi$ & $\varphi_{\exp }$ & osmotic pressure & calculated $\varphi$ & $\varphi_{\exp }$ & osmotic pressure & calculated $\varphi$ & $\varphi_{\exp }$ \\
\hline 0.2 & $19.0 \pm 1.1$ & $1.00 \pm 0.06$ & 0.80 & $17.0 \pm 1.1$ & $0.90 \pm 0.06$ & 0.80 & $15.9 \pm 1.7$ & $0.83 \pm 0.09$ & 0.81 \\
\hline 0.5 & $41.8 \pm 1.4$ & $0.93 \pm 0.03$ & 0.89 & $41.2 \pm 1.6$ & $0.92 \pm 0.04$ & 0.89 & $39.7 \pm 1.8$ & $0.86 \pm 0.04$ & 0.92 \\
\hline 1.5 & $149.5 \pm 3.6$ & $1.26 \pm 0.03$ & 1.27 & $151.5 \pm 4.2$ & $1.27 \pm 0.04$ & 1.26 & $142.3 \pm 8.9$ & $1.15 \pm 0.07$ & 1.39 \\
\hline 2.0 & $238.6 \pm 3.5$ & $1.59 \pm 0.02$ & 1.47 & $235.8 \pm 4.1$ & $1.56 \pm 0.03$ & 1.46 & $224.3 \pm 8.5$ & $1.42 \pm 0.05$ & 1.64 \\
\hline 2.5 & $289.7 \pm 9.2$ & $1.61 \pm 0.05$ & 1.67 & $294.0 \pm 8.0$ & $1.63 \pm 0.04$ & 1.66 & $303.0 \pm 7.5$ & $1.59 \pm 0.04$ & 1.90 \\
\hline
\end{tabular}


Table S7. Calculated Osmotic Pressure (bar) and Osmotic Coefficient of $\mathrm{AlCl}_{3}$ Solution Using LJ Parameters between $\mathrm{Al}^{3+}$ and $\mathrm{Cl}^{-} \mathrm{Derived}$ from the Lorentz-Berthelot Mixing Rule

\begin{tabular}{|c|c|c|c|c|c|c|}
\hline \multirow[t]{2}{*}{ molarity } & \multicolumn{2}{|c|}{ TIP3P } & \multicolumn{2}{|c|}{$\mathrm{SPC} / \mathrm{E}$} & \multicolumn{2}{|c|}{ TIP4P-Ew } \\
\hline & osmotic pressure & osmotic coefficient & osmotic pressure & osmotic coefficient & osmotic pressure & osmotic coefficient \\
\hline 0.2 & $13.4 \pm 1.0$ & $0.69 \pm 0.05$ & $12.9 \pm 1.2$ & $0.67 \pm 0.06$ & $10.9 \pm 0.8$ & $0.56 \pm 0.04$ \\
\hline 0.5 & $24.3 \pm 2.5$ & $0.52 \pm 0.05$ & $31.1 \pm 2.6$ & $0.66 \pm 0.06$ & $26.7 \pm 1.4$ & $0.57 \pm 0.03$ \\
\hline 1.0 & $37.4 \pm 2.1$ & $0.41 \pm 0.02$ & $73.2 \pm 4.9$ & $0.81 \pm 0.05$ & $41.8 \pm 2.4$ & $0.46 \pm 0.03$ \\
\hline 1.5 & $46.8 \pm 1.8$ & $0.36 \pm 0.01$ & $118.0 \pm 5.1$ & $0.90 \pm 0.04$ & $65.1 \pm 3.7$ & $0.50 \pm 0.03$ \\
\hline 2.0 & $43.5 \pm 2.4$ & $0.26 \pm 0.01$ & $132.5 \pm 3.2$ & $0.78 \pm 0.02$ & $81.2 \pm 6.4$ & $0.48 \pm 0.04$ \\
\hline 2.5 & $47.5 \pm 3.0$ & $0.23 \pm 0.02$ & $188.0 \pm 6.2$ & $0.91 \pm 0.03$ & $75.0 \pm 5.0$ & $0.36 \pm 0.02$ \\
\hline 3.0 & $52.9 \pm 2.6$ & $0.22 \pm 0.01$ & $236.0 \pm 5.5$ & $0.98 \pm 0.02$ & $106.4 \pm 10.2$ & $0.44 \pm 0.04$ \\
\hline
\end{tabular}


Table S8. Calculated Osmotic Pressure (bar) and Osmotic Coefficient ( $\varphi$ ) of $\mathrm{AlCl}_{3}$ Solution Using Different Methods of Handling vdW Interactions and Different $\mathrm{LJ}$ Parameters between $\mathrm{Al}^{3+}$ and $\mathrm{Cl}^{-a}$

\begin{tabular}{|c|c|c|c|c|c|c|c|}
\hline \multirow[t]{2}{*}{ molarity } & \multicolumn{2}{|c|}{ LB mixing rule ${ }^{b}$ (cut-off) } & \multicolumn{2}{|c|}{ modified LJ, set $1^{b}$ (cut-off) } & \multicolumn{2}{|c|}{ modified LJ, set $2^{b}$ (cut-off) } & \multirow[b]{2}{*}{$\varphi^{\exp }$} \\
\hline & osmotic pressure & calculated $\varphi$ & osmotic pressure & calculated $\varphi \mathrm{t}$ & osmotic pressure & calculated $\varphi$ & \\
\hline 0.2 & $13.4 \pm 1.0$ & $0.69 \pm 0.05$ & $17.0 \pm 0.8$ & $0.88 \pm 0.04$ & $18.8 \pm 0.9$ & $0.97 \pm 0.05$ & 0.85 \\
\hline 0.5 & $24.3 \pm 2.5$ & $0.52 \pm 0.05$ & $46.9 \pm 1.7$ & $1.00 \pm 0.04$ & $46.4 \pm 2.6$ & $0.98 \pm 0.06$ & 0.98 \\
\hline 1.0 & $37.4 \pm 2.1$ & $0.41 \pm 0.02$ & $130.2 \pm 2.4$ & $1.44 \pm 0.03$ & $121.0 \pm 4.1$ & $1.34 \pm 0.05$ & 1.30 \\
\hline 1.5 & $46.8 \pm 1.8$ & $0.36 \pm 0.01$ & $225.3 \pm 4.8$ & $1.72 \pm 0.04$ & $228.2 \pm 6.9$ & $1.74 \pm 0.05$ & 1.67 \\
\hline 2.0 & $43.5 \pm 2.4$ & $0.26 \pm 0.01$ & $362.8 \pm 3.9$ & $2.14 \pm 0.02$ & $363.1 \pm 4.8$ & $2.15 \pm 0.03$ & 2.08 \\
\hline 2.5 & $47.5 \pm 3.0$ & $0.23 \pm 0.02$ & $518.6 \pm 5.6$ & $2.52 \pm 0.03$ & $565.4 \pm 9.1$ & $2.75 \pm 0.04$ & 2.55 \\
\hline 3.0 & $52.9 \pm 2.6$ & $0.22 \pm 0.01$ & $726.7 \pm 9.8$ & $3.02 \pm 0.04$ & $724.6 \pm 10.6$ & $3.01 \pm 0.04$ & 3.02 \\
\hline RMSE & & 1.66 & & 0.06 & & 0.10 & \\
\hline MAE & & 1.40 & & 0.05 & & 0.07 & \\
\hline MSE & & -1.40 & & 0.04 & & 0.07 & \\
\hline \multirow[t]{2}{*}{ molarity } & \multicolumn{2}{|c|}{ LB mixing rule ${ }^{b}$ (LJ-PME) } & \multicolumn{2}{|c|}{ modified LJ, set $1^{b}$ (LJ-PME) } & \multicolumn{2}{|c|}{ modified LJ, set $2^{b}$ (LJ-PME) } & \\
\hline & osmotic pressure & calculated $\varphi$ & osmotic pressure & calculated $\varphi$ & osmotic pressure & calculated $\varphi$ & $\varphi^{\exp }$ \\
\hline 0.2 & $14.2+/-0.9$ & $0.73+/-0.05$ & $19.3+/-0.8$ & $1.00+/-0.04$ & $20.3+/-1.2$ & $1.05+/-0.06$ & 0.85 \\
\hline 0.5 & $25.2+/-2.0$ & $0.54+/-0.04$ & $49.6+/-1.7$ & $1.05+/-0.04$ & $48.4+/-1.4$ & $1.03+/-0.03$ & 0.98 \\
\hline 1.0 & $41.7+/-2.5$ & $0.46+/-0.03$ & $119.1+/-3.1$ & $1.32+/-0.03$ & $128.1+/-3.2$ & $1.42+/-0.04$ & 1.30 \\
\hline 1.5 & $43.4+/-2.1$ & $0.33+/-0.02$ & $225.9+/-3.7$ & $1.73+/-0.03$ & $220.7+/-4.5$ & $1.69+/-0.03$ & 1.67 \\
\hline 2.0 & $42.8+/-2.1$ & $0.25+/-0.01$ & $363.5+/-7.2$ & $2.15+/-0.04$ & $375.9+/-4.2$ & $2.22+/-0.03$ & 2.08 \\
\hline 2.5 & $36.7+/-2.4$ & $0.18+/-0.01$ & $531.5+/-5.3$ & $2.58+/-0.03$ & $534.8+/-7.6$ & $2.60+/-0.04$ & 2.55 \\
\hline 3.0 & $53.8+/-3.0$ & $0.22+/-0.01$ & $702.4+/-14.8$ & $2.92+/-0.06$ & $765.0+/-11.9$ & $3.17+/-0.05$ & 3.02 \\
\hline RMSE & & 1.67 & & 0.08 & & 0.12 & \\
\hline MAE & & 1.40 & & 0.07 & & 0.10 & \\
\hline MSE & & -1.40 & & 0.04 & & 0.10 & \\
\hline
\end{tabular}


${ }^{a}$ The methods of handling vdW interactions are the cut-off scheme with a dispersion correction of energy and pressure and the Lennard-Jones PME (LJ-PME) scheme. ${ }^{6,7}$ Experimental osmotic coefficient $\left(\varphi_{\text {exp }}\right)$ were taken from polynomial fits of data points in ref. ${ }^{16,17 b} b J$ parameters are given in Table 7 in the main text. 
Table S9. Final Optimized LJ Parameters for TIP3P Water Model in Different 12-6 LJ

\section{Forms}

\begin{tabular}{|c|c|c|c|c|c|c|}
\hline Ion & $\begin{array}{c}\mathrm{C}_{6} \\
\left(\mathrm{~kJ} \mathrm{~mol}^{-1} \mathrm{~nm}^{6}\right)\end{array}$ & $\begin{array}{c}\mathrm{C}_{12} \\
\left(\mathrm{~kJ} \mathrm{~mol}^{-1} \mathrm{~nm}^{12}\right)\end{array}$ & $\begin{array}{l}\text { sigma } \\
(\mathrm{nm})\end{array}$ & $\begin{array}{l}\text { epsilon } \\
(\mathrm{kJ} / \mathrm{mol})\end{array}$ & $\begin{array}{c}R_{\min } / 2 \\
(\AA)\end{array}$ & $\begin{array}{c}\text { epsilon } \\
(\mathrm{kcal} / \mathrm{mol})\end{array}$ \\
\hline $\mathrm{Al}^{3+}$ & $1.29634 \mathrm{e}-04$ & $3.76602 \mathrm{e}-13$ & $3.77741 \mathrm{e}-02$ & $1.11556 \mathrm{e}+04$ & 0.2120 & 2666.2447 \\
\hline $\mathrm{Cr}^{3+}$ & $8.16641 \mathrm{e}-04$ & $9.59864 \mathrm{e}-11$ & $6.99890 \mathrm{e}-02$ & $1.73697 \mathrm{e}+03$ & 0.3928 & 415.1452 \\
\hline $\mathrm{Fe}^{3+}$ & $1.69295 \mathrm{e}-03$ & $1.00101 \mathrm{e}-10$ & $6.24164 \mathrm{e}-02$ & $7.15800 \mathrm{e}+03$ & 0.3503 & 1710.8030 \\
\hline $\operatorname{In}^{3+}$ & $4.45848 \mathrm{e}-03$ & $1.96029 \mathrm{e}-09$ & $8.72012 \mathrm{e}-02$ & $2.53508 \mathrm{e}+03$ & 0.4894 & 605.8987 \\
\hline $\mathrm{Lu}^{3+}$ & $1.55848 \mathrm{e}-02$ & $1.76706 \mathrm{e}-07$ & $1.49885 \mathrm{e}-01$ & $3.43631 \mathrm{e}+02$ & 0.8412 & 82.1297 \\
\hline $\mathrm{Tl}^{3+}$ & $1.62450 \mathrm{e}-02$ & $1.89055 \mathrm{e}-08$ & $1.02560 \mathrm{e}-01$ & $3.48973 \mathrm{e}+03$ & 0.5756 & 834.0651 \\
\hline $\mathrm{Tm}^{3+}$ & $1.98140 \mathrm{e}-02$ & $2.33449 \mathrm{e}-07$ & $1.50847 \mathrm{e}-01$ & $4.20428 \mathrm{e}+02$ & 0.8466 & 100.4847 \\
\hline $\mathrm{Er}^{3+}$ & $1.97548 \mathrm{e}-02$ & $2.62782 \mathrm{e}-07$ & $1.53929 \mathrm{e}-01$ & $3.71271 \mathrm{e}+02$ & 0.8639 & 88.7360 \\
\hline $\mathrm{Y}^{3+}$ & $1.74621 \mathrm{e}-02$ & $2.99943 \mathrm{e}-07$ & $1.60629 \mathrm{e}-01$ & $2.54151 \mathrm{e}+02$ & 0.9015 & 60.7435 \\
\hline $\mathrm{Dy}^{3+}$ & $1.56761 \mathrm{e}-02$ & $3.15446 \mathrm{e}-07$ & $1.64923 \mathrm{e}-01$ & $1.94756 \mathrm{e}+02$ & 0.9256 & 46.5479 \\
\hline $\mathrm{Tb}^{3+}$ & $2.16391 \mathrm{e}-02$ & $5.12893 \mathrm{e}-07$ & $1.69485 \mathrm{e}-01$ & $2.28240 \mathrm{e}+02$ & 0.9512 & 54.5507 \\
\hline $\mathrm{Gd}^{3+}$ & $1.56968 \mathrm{e}-02$ & $4.56528 \mathrm{e}-07$ & $1.75365 \mathrm{e}-01$ & $1.34925 \mathrm{e}+02$ & 0.9842 & 32.2478 \\
\hline $\mathrm{Eu}^{3+}$ & $3.32580 \mathrm{e}-02$ & $1.00379 \mathrm{e}-06$ & $1.76451 \mathrm{e}-01$ & $2.75481 \mathrm{e}+02$ & 0.9903 & 65.8415 \\
\hline $\mathrm{Sm}^{3+}$ & $2.84776 \mathrm{e}-02$ & $1.06673 \mathrm{e}-06$ & $1.82919 \mathrm{e}-01$ & $1.90060 \mathrm{e}+02$ & 1.0266 & 45.4255 \\
\hline $\mathrm{Nd}^{3+}$ & $3.30341 \mathrm{e}-02$ & $1.56342 \mathrm{e}-06$ & $1.90189 \mathrm{e}-01$ & $1.74498 \mathrm{e}+02$ & 1.0674 & 41.7061 \\
\hline $\operatorname{Pr}^{3+}$ & $5.48259 \mathrm{e}-02$ & $3.13705 \mathrm{e}-06$ & $1.96301 \mathrm{e}-01$ & $2.39547 \mathrm{e}+02$ & 1.1017 & 57.2532 \\
\hline $\mathrm{Ce}^{3+}$ & $4.80105 \mathrm{e}-02$ & $3.52225 \mathrm{e}-06$ & $2.04604 \mathrm{e}-01$ & $1.63603 \mathrm{e}+02$ & 1.1483 & 39.1021 \\
\hline $\mathrm{La}^{3+}$ & $2.50640 \mathrm{e}-02$ & $2.83889 \mathrm{e}-06$ & $2.19963 \mathrm{e}-01$ & $5.53213 \mathrm{e}+01$ & 1.2345 & 13.2221 \\
\hline $\mathrm{Hf}^{4+}$ & $2.18405 \mathrm{e}-02$ & $7.25895 \mathrm{e}-09$ & $8.32278 \mathrm{e}-02$ & $1.64283 \mathrm{e}+04$ & 0.4671 & 3926.4494 \\
\hline $\mathrm{Zr}^{4+}$ & $2.59651 \mathrm{e}-02$ & $1.41622 \mathrm{e}-08$ & $9.03906 \mathrm{e}-02$ & $1.19012 \mathrm{e}+04$ & 0.5073 & 2844.4611 \\
\hline $\mathrm{Ce}^{4+}$ & $1.40402 \mathrm{e}-01$ & $1.17868 \mathrm{e}-06$ & $1.42562 \mathrm{e}-01$ & $4.18111 \mathrm{e}+03$ & 0.8001 & 999.3095 \\
\hline $\mathrm{Pu}^{4+}$ & $1.77951 \mathrm{e}-01$ & $7.15400 \mathrm{e}-07$ & $1.26098 \mathrm{e}-01$ & $1.10660 \mathrm{e}+04$ & 0.7077 & 2644.8436 \\
\hline $\mathrm{U}^{4+}$ & $2.07466 \mathrm{e}-01$ & $1.34847 \mathrm{e}-06$ & $1.36610 \mathrm{e}-01$ & $7.97979 \mathrm{e}+03$ & 0.7667 & 1907.2165 \\
\hline $\mathrm{Th}^{4+}$ & $1.24269 \mathrm{e}-01$ & $2.16310 \mathrm{e}-06$ & $1.60985 \mathrm{e}-01$ & $1.78481 \mathrm{e}+03$ & 0.9035 & 426.5795 \\
\hline
\end{tabular}


Table S10. Final Optimized LJ Parameters for OPC3 Water Model in Different 12-6 LJ

\section{Forms}

\begin{tabular}{|c|c|c|c|c|c|c|}
\hline Ion & $\begin{array}{c}\mathrm{C}_{6} \\
\left(\mathrm{~kJ} \mathrm{~mol}^{-1} \mathrm{~nm}^{6}\right)\end{array}$ & $\begin{array}{c}\mathrm{C}_{12} \\
\left(\mathrm{~kJ} \mathrm{~mol}^{-1} \mathrm{~nm}^{12}\right)\end{array}$ & $\begin{array}{l}\text { sigma } \\
(\mathrm{nm})\end{array}$ & $\begin{array}{l}\text { epsilon } \\
(\mathrm{kJ} / \mathrm{mol})\end{array}$ & $\begin{array}{c}R_{\min } / 2 \\
(\AA)\end{array}$ & $\begin{array}{c}\text { epsilon } \\
(\mathrm{kcal} / \mathrm{mol})\end{array}$ \\
\hline $\mathrm{Al}^{3+}$ & $1.14492 \mathrm{e}-04$ & $4.66553 \mathrm{e}-13$ & $3.99657 \mathrm{e}-02$ & $7.02412 \mathrm{e}+03$ & 0.2243 & 1678.8040 \\
\hline $\mathrm{Cr}^{3+}$ & $6.60009 \mathrm{e}-04$ & $1.60934 \mathrm{e}-10$ & $7.90405 \mathrm{e}-02$ & $6.76693 \mathrm{e}+02$ & 0.4436 & 161.7335 \\
\hline $\mathrm{Fe}^{3+}$ & $1.50533 \mathrm{e}-03$ & $1.13530 \mathrm{e}-10$ & $6.50000 \mathrm{e}-02$ & $4.98990 \mathrm{e}+03$ & 0.3648 & 1192.6143 \\
\hline $\operatorname{In}^{3+}$ & $2.88669 \mathrm{e}-03$ & $1.41075 \mathrm{e}-09$ & $8.87513 \mathrm{e}-02$ & $1.47670 \mathrm{e}+03$ & 0.4981 & 352.9393 \\
\hline $\mathrm{Lu}^{3+}$ & $1.17092 \mathrm{e}-02$ & $1.99391 \mathrm{e}-07$ & $1.60397 \mathrm{e}-01$ & $1.71906 \mathrm{e}+02$ & 0.9002 & 41.0866 \\
\hline $\mathrm{Tl}^{3+}$ & $1.26364 \mathrm{e}-02$ & $1.73178 \mathrm{e}-08$ & $1.05393 \mathrm{e}-01$ & $2.30511 \mathrm{e}+03$ & 0.5915 & 550.9345 \\
\hline $\operatorname{Tm}^{3+}$ & $1.50580 \mathrm{e}-02$ & $2.54379 \mathrm{e}-07$ & $1.60184 \mathrm{e}-01$ & $2.22839 \mathrm{e}+02$ & 0.8990 & 53.2599 \\
\hline $\mathrm{Er}^{3+}$ & $1.40090 \mathrm{e}-02$ & $2.70934 \mathrm{e}-07$ & $1.63836 \mathrm{e}-01$ & $1.81089 \mathrm{e}+02$ & 0.9195 & 43.2813 \\
\hline $\mathrm{Y}^{3+}$ & $1.10815 \mathrm{e}-02$ & $3.05948 \mathrm{e}-07$ & $1.73850 \mathrm{e}-01$ & $1.00344 \mathrm{e}+02$ & 0.9757 & 23.9828 \\
\hline $\mathrm{Dy}^{3+}$ & $1.06434 \mathrm{e}-02$ & $3.50516 \mathrm{e}-07$ & $1.79035 \mathrm{e}-01$ & $8.07964 \mathrm{e}+01$ & 1.0048 & 19.3108 \\
\hline $\mathrm{Tb}^{3+}$ & $1.44174 \mathrm{e}-02$ & $5.31277 \mathrm{e}-07$ & $1.82420 \mathrm{e}-01$ & $9.78119 \mathrm{e}+01$ & 1.0238 & 23.3776 \\
\hline $\mathrm{Gd}^{3+}$ & $7.87610 \mathrm{e}-03$ & $4.33978 \mathrm{e}-07$ & $1.95071 \mathrm{e}-01$ & $3.57351 \mathrm{e}+01$ & 1.0948 & 8.5409 \\
\hline $\mathrm{Eu}^{3+}$ & $2.31702 \mathrm{e}-02$ & $1.00788 \mathrm{e}-06$ & $1.87534 \mathrm{e}-01$ & $1.33165 \mathrm{e}+02$ & 1.0525 & 31.8273 \\
\hline $\mathrm{Sm}^{3+}$ & $1.42554 \mathrm{e}-02$ & $8.89839 \mathrm{e}-07$ & $1.99169 \mathrm{e}-01$ & $5.70940 \mathrm{e}+01$ & 1.1178 & 13.6458 \\
\hline $\mathrm{Nd}^{3+}$ & $1.66633 \mathrm{e}-02$ & $1.33037 \mathrm{e}-06$ & $2.07508 \mathrm{e}-01$ & $5.21787 \mathrm{e}+01$ & 1.1646 & 12.4710 \\
\hline $\operatorname{Pr}^{3+}$ & $3.28908 \mathrm{e}-02$ & $2.77266 \mathrm{e}-06$ & $2.09397 \mathrm{e}-01$ & $9.75420 \mathrm{e}+01$ & 1.1752 & 23.3131 \\
\hline $\mathrm{Ce}^{3+}$ & $2.87950 \mathrm{e}-02$ & $3.20171 \mathrm{e}-06$ & $2.19286 \mathrm{e}-01$ & $6.47428 \mathrm{e}+01$ & 1.2307 & 15.4739 \\
\hline $\mathrm{La}^{3+}$ & $1.47298 \mathrm{e}-02$ & $2.87367 \mathrm{e}-06$ & $2.40828 \mathrm{e}-01$ & $1.88753 \mathrm{e}+01$ & 1.3516 & 4.5113 \\
\hline $\mathrm{Hf}^{4+}$ & $1.82346 \mathrm{e}-02$ & $6.90781 \mathrm{e}-09$ & $8.50630 \mathrm{e}-02$ & $1.20335 \mathrm{e}+04$ & 0.4774 & 2876.0736 \\
\hline $\mathrm{Zr}^{4+}$ & $2.29287 \mathrm{e}-02$ & $1.52408 \mathrm{e}-08$ & $9.34196 \mathrm{e}-02$ & $8.62366 \mathrm{e}+03$ & 0.5243 & 2061.1045 \\
\hline $\mathrm{Ce}^{4+}$ & $9.94288 \mathrm{e}-02$ & $9.27626 \mathrm{e}-07$ & $1.45092 \mathrm{e}-01$ & $2.66435 \mathrm{e}+03$ & 0.8143 & 636.7955 \\
\hline $\mathrm{Pu}^{4+}$ & $1.41866 \mathrm{e}-01$ & $6.06049 \mathrm{e}-07$ & $1.27381 \mathrm{e}-01$ & $8.30217 \mathrm{e}+03$ & 0.7149 & 1984.2665 \\
\hline $\mathrm{U}^{4+}$ & $1.77059 \mathrm{e}-01$ & $1.32582 \mathrm{e}-06$ & $1.39871 \mathrm{e}-01$ & $5.91142 \mathrm{e}+03$ & 0.7850 & 1412.8628 \\
\hline $\mathrm{Th}^{4+}$ & $1.61408 \mathrm{e}-01$ & $4.24408 \mathrm{e}-06$ & $1.72442 \mathrm{e}-01$ & $1.53464 \mathrm{e}+03$ & 0.9678 & 366.7874 \\
\hline
\end{tabular}


Table S11. Final Optimized LJ Parameters for SPC/E Water Model in Different 12-6

\section{LJ Forms}

\begin{tabular}{|c|c|c|c|c|c|c|}
\hline Ion & $\begin{array}{c}\mathrm{C}_{6} \\
\left(\mathrm{~kJ} \mathrm{~mol}^{-1} \mathrm{~nm}^{6}\right)\end{array}$ & $\begin{array}{c}\mathrm{C}_{12} \\
\left(\mathrm{~kJ} \mathrm{~mol}^{-1} \mathrm{~nm}^{12}\right)\end{array}$ & $\begin{array}{l}\text { sigma } \\
(\mathrm{nm})\end{array}$ & $\begin{array}{l}\text { epsilon } \\
(\mathrm{kJ} / \mathrm{mol})\end{array}$ & $\begin{array}{c}R_{\min } / 2 \\
(\AA)\end{array}$ & $\begin{array}{c}\text { epsilon } \\
(\mathrm{kcal} / \mathrm{mol})\end{array}$ \\
\hline $\mathrm{Al}^{3+}$ & $1.33890 \mathrm{e}-04$ & $4.58609 \mathrm{e}-13$ & $3.88254 \mathrm{e}-02$ & $9.77218 \mathrm{e}+03$ & 0.2179 & 2335.6082 \\
\hline $\mathrm{Cr}^{3+}$ & $7.55991 \mathrm{e}-04$ & $1.08189 \mathrm{e}-10$ & $7.23232 \mathrm{e}-02$ & $1.32066 \mathrm{e}+03$ & 0.4059 & 315.6458 \\
\hline $\mathrm{Fe}^{3+}$ & $1.56939 \mathrm{e}-03$ & $9.31125 \mathrm{e}-11$ & $6.24520 \mathrm{e}-02$ & $6.61290 \mathrm{e}+03$ & 0.3505 & 1580.5201 \\
\hline $\operatorname{In}^{3+}$ & $3.78913 \mathrm{e}-03$ & $1.61558 \mathrm{e}-09$ & $8.67557 \mathrm{e}-02$ & $2.22173 \mathrm{e}+03$ & 0.4869 & 531.0067 \\
\hline $\mathrm{Lu}^{3+}$ & $1.47852 \mathrm{e}-02$ & $1.80844 \mathrm{e}-07$ & $1.51791 \mathrm{e}-01$ & $3.02198 \mathrm{e}+02$ & 0.8519 & 72.2271 \\
\hline $\mathrm{Tl}^{3+}$ & $1.45544 \mathrm{e}-02$ & $1.71332 \mathrm{e}-08$ & $1.02756 \mathrm{e}-01$ & $3.09095 \mathrm{e}+03$ & 0.5767 & 738.7543 \\
\hline $\operatorname{Tm}^{3+}$ & $1.80781 \mathrm{e}-02$ & $2.28062 \mathrm{e}-07$ & $1.52575 \mathrm{e}-01$ & $3.58255 \mathrm{e}+02$ & 0.8563 & 85.6249 \\
\hline $\mathrm{Er}^{3+}$ & $1.77819 \mathrm{e}-02$ & $2.52583 \mathrm{e}-07$ & $1.55622 \mathrm{e}-01$ & $3.12962 \mathrm{e}+02$ & 0.8734 & 74.7997 \\
\hline $\mathrm{Y}^{3+}$ & $1.47411 \mathrm{e}-02$ & $2.77913 \mathrm{e}-07$ & $1.63141 \mathrm{e}-01$ & $1.95475 \mathrm{e}+02$ & 0.9156 & 46.7197 \\
\hline $\mathrm{Dy}^{3+}$ & $1.29196 \mathrm{e}-02$ & $2.96309 \mathrm{e}-07$ & $1.68558 \mathrm{e}-01$ & $1.40829 \mathrm{e}+02$ & 0.9460 & 33.6589 \\
\hline $\mathrm{Tb}^{3+}$ & $1.78330 \mathrm{e}-02$ & $4.78295 \mathrm{e}-07$ & $1.73013 \mathrm{e}-01$ & $1.66223 \mathrm{e}+02$ & 0.9710 & 39.7283 \\
\hline $\mathrm{Gd}^{3+}$ & $1.24611 \mathrm{e}-02$ & $4.15298 \mathrm{e}-07$ & $1.79391 \mathrm{e}-01$ & $9.34743 \mathrm{e}+01$ & 1.0068 & 22.3409 \\
\hline $\mathrm{Eu}^{3+}$ & $3.13982 \mathrm{e}-02$ & $1.06086 \mathrm{e}-06$ & $1.79801 \mathrm{e}-01$ & $2.32323 \mathrm{e}+02$ & 1.0091 & 55.5265 \\
\hline $\mathrm{Sm}^{3+}$ & $2.54491 \mathrm{e}-02$ & $1.07645 \mathrm{e}-06$ & $1.86661 \mathrm{e}-01$ & $1.50415 \mathrm{e}+02$ & 1.0476 & 35.9501 \\
\hline $\mathrm{Nd}^{3+}$ & $2.85497 \mathrm{e}-02$ & $1.53304 \mathrm{e}-06$ & $1.94234 \mathrm{e}-01$ & $1.32920 \mathrm{e}+02$ & 1.0901 & 31.7687 \\
\hline $\operatorname{Pr}^{3+}$ & $5.12009 \mathrm{e}-02$ & $3.13981 \mathrm{e}-06$ & $1.98581 \mathrm{e}-01$ & $2.08733 \mathrm{e}+02$ & 1.1145 & 49.8884 \\
\hline $\mathrm{Ce}^{3+}$ & $4.20285 \mathrm{e}-02$ & $3.34684 \mathrm{e}-06$ & $2.07419 \mathrm{e}-01$ & $1.31945 \mathrm{e}+02$ & 1.1641 & 31.5355 \\
\hline $\mathrm{La}^{3+}$ & $1.84065 \mathrm{e}-02$ & $2.48307 \mathrm{e}-06$ & $2.26466 \mathrm{e}-01$ & $3.41109 \mathrm{e}+01$ & 1.2710 & 8.1527 \\
\hline $\mathrm{Hf}^{4+}$ & $1.80769 \mathrm{e}-02$ & $5.61045 \mathrm{e}-09$ & $8.22834 \mathrm{e}-02$ & $1.45610 \mathrm{e}+04$ & 0.4618 & 3480.1663 \\
\hline $\mathrm{Zr}^{4+}$ & $2.23684 \mathrm{e}-02$ & $1.18582 \mathrm{e}-08$ & $8.99630 \mathrm{e}-02$ & $1.05485 \mathrm{e}+04$ & 0.5049 & 2521.1576 \\
\hline $\mathrm{Ce}^{4+}$ & $1.27959 \mathrm{e}-01$ & $1.10025 \mathrm{e}-06$ & $1.43132 \mathrm{e}-01$ & $3.72042 \mathrm{e}+03$ & 0.8033 & 889.2011 \\
\hline $\mathrm{Pu}^{4+}$ & $1.54802 \mathrm{e}-01$ & $6.00485 \mathrm{e}-07$ & $1.25349 \mathrm{e}-01$ & $9.97681 \mathrm{e}+03$ & 0.7035 & 2384.5147 \\
\hline $\mathrm{U}^{4+}$ & $1.90225 \mathrm{e}-01$ & $1.24906 \mathrm{e}-06$ & $1.36842 \mathrm{e}-01$ & $7.24255 \mathrm{e}+03$ & 0.7680 & 1731.0116 \\
\hline $\mathrm{Th}^{4+}$ & $1.53342 \mathrm{e}-01$ & $3.16865 \mathrm{e}-06$ & $1.65654 \mathrm{e}-01$ & $1.85520 \mathrm{e}+03$ & 0.9297 & 443.4044 \\
\hline
\end{tabular}


Table S12. Final Optimized LJ Parameters for $\mathrm{SPC} / \mathrm{E}_{\mathrm{b}}$ Water Model in Different 12-6

\section{LJ Forms}

\begin{tabular}{|c|c|c|c|c|c|c|}
\hline Ion & $\begin{array}{c}\mathrm{C}_{6} \\
\left(\mathrm{~kJ} \mathrm{~mol}{ }^{-1} \mathrm{~nm}^{6}\right)\end{array}$ & $\begin{array}{c}\mathrm{C}_{12} \\
\left(\mathrm{~kJ} \mathrm{~mol}^{-1} \mathrm{~nm}^{12}\right)\end{array}$ & $\begin{array}{l}\text { sigma } \\
(\mathrm{nm})\end{array}$ & $\begin{array}{l}\text { epsilon } \\
(\mathrm{kJ} / \mathrm{mol})\end{array}$ & $\begin{array}{c}R_{\min } / 2 \\
(\AA)\end{array}$ & $\begin{array}{c}\text { epsilon } \\
(\mathrm{kcal} / \mathrm{mol})\end{array}$ \\
\hline $\mathrm{Al}^{3+}$ & $1.35905 \mathrm{e}-04$ & $4.93081 \mathrm{e}-13$ & $3.91995 \mathrm{e}-02$ & $9.36465 \mathrm{e}+03$ & 0.2200 & 2238.2057 \\
\hline $\mathrm{Cr}^{3+}$ & $8.16292 \mathrm{e}-04$ & $1.33825 \mathrm{e}-10$ & $7.39802 \mathrm{e}-02$ & $1.24478 \mathrm{e}+03$ & 0.4152 & 297.5089 \\
\hline $\mathrm{Fe}^{3+}$ & $1.63042 \mathrm{e}-03$ & $1.03549 \mathrm{e}-10$ & $6.31647 \mathrm{e}-02$ & $6.41788 \mathrm{e}+03$ & 0.3545 & 1533.9104 \\
\hline $\operatorname{In}^{3+}$ & $3.45561 \mathrm{e}-03$ & $1.39700 \mathrm{e}-09$ & $8.59895 \mathrm{e}-02$ & $2.13694 \mathrm{e}+03$ & 0.4826 & 510.7402 \\
\hline $\mathrm{Lu}^{3+}$ & $1.36326 \mathrm{e}-02$ & $1.75755 \mathrm{e}-07$ & $1.53128 \mathrm{e}-01$ & $2.64358 \mathrm{e}+02$ & 0.8594 & 63.1830 \\
\hline $\mathrm{Tl}^{3+}$ & $1.68834 \mathrm{e}-02$ & $2.26499 \mathrm{e}-08$ & $1.05019 \mathrm{e}-01$ & $3.14624 \mathrm{e}+03$ & 0.5894 & 751.9691 \\
\hline $\operatorname{Tm}^{3+}$ & $1.70628 \mathrm{e}-02$ & $2.24621 \mathrm{e}-07$ & $1.53662 \mathrm{e}-01$ & $3.24035 \mathrm{e}+02$ & 0.8624 & 77.4462 \\
\hline $\mathrm{Er}^{3+}$ & $1.64124 \mathrm{e}-02$ & $2.44902 \mathrm{e}-07$ & $1.56905 \mathrm{e}-01$ & $2.74974 \mathrm{e}+02$ & 0.8806 & 65.7204 \\
\hline $\mathrm{Y}^{3+}$ & $1.39810 \mathrm{e}-02$ & $2.77347 \mathrm{e}-07$ & $1.64531 \mathrm{e}-01$ & $1.76194 \mathrm{e}+02$ & 0.9234 & 42.1114 \\
\hline $\mathrm{Dy}^{3+}$ & $1.35908 \mathrm{e}-02$ & $3.19699 \mathrm{e}-07$ & $1.69271 \mathrm{e}-01$ & $1.44441 \mathrm{e}+02$ & 0.9500 & 34.5223 \\
\hline $\mathrm{Tb}^{3+}$ & $1.89664 \mathrm{e}-02$ & $5.17866 \mathrm{e}-07$ & $1.73529 \mathrm{e}-01$ & $1.73657 \mathrm{e}+02$ & 0.9739 & 41.5050 \\
\hline $\mathrm{Gd}^{3+}$ & $1.49889 \mathrm{e}-02$ & $4.85722 \mathrm{e}-07$ & $1.78554 \mathrm{e}-01$ & $1.15636 \mathrm{e}+02$ & 1.0021 & 27.6376 \\
\hline $\mathrm{Eu}^{3+}$ & $3.22736 \mathrm{e}-02$ & $1.11005 \mathrm{e}-06$ & $1.80336 \mathrm{e}-01$ & $2.34581 \mathrm{e}+02$ & 1.0121 & 56.0661 \\
\hline $\mathrm{Sm}^{3+}$ & $2.72734 \mathrm{e}-02$ & $1.14770 \mathrm{e}-06$ & $1.86501 \mathrm{e}-01$ & $1.62029 \mathrm{e}+02$ & 1.0467 & 38.7258 \\
\hline $\mathrm{Nd}^{3+}$ & $2.91879 \mathrm{e}-02$ & $1.56731 \mathrm{e}-06$ & $1.94234 \mathrm{e}-01$ & $1.35891 \mathrm{e}+02$ & 1.0901 & 32.4788 \\
\hline $\operatorname{Pr}^{3+}$ & $5.01312 \mathrm{e}-02$ & $3.18173 \mathrm{e}-06$ & $1.99722 \mathrm{e}-01$ & $1.97466 \mathrm{e}+02$ & 1.1209 & 47.1954 \\
\hline $\mathrm{Ce}^{3+}$ & $3.88090 \mathrm{e}-02$ & $3.22339 \mathrm{e}-06$ & $2.08880 \mathrm{e}-01$ & $1.16813 \mathrm{e}+02$ & 1.1723 & 27.9190 \\
\hline $\mathrm{La}^{3+}$ & $2.35566 \mathrm{e}-02$ & $2.97355 \mathrm{e}-06$ & $2.23972 \mathrm{e}-01$ & $4.66541 \mathrm{e}+01$ & 1.2570 & 11.1506 \\
\hline $\mathrm{Hf}^{4+}$ & $1.72256 \mathrm{e}-02$ & $5.29780 \mathrm{e}-09$ & $8.21587 \mathrm{e}-02$ & $1.40021 \mathrm{e}+04$ & 0.4611 & 3346.5707 \\
\hline $\mathrm{Zr}^{4+}$ & $2.40246 \mathrm{e}-02$ & $1.37360 \mathrm{e}-08$ & $9.11033 \mathrm{e}-02$ & $1.05049 \mathrm{e}+04$ & 0.5113 & 2510.7299 \\
\hline $\mathrm{Ce}^{4+}$ & $1.23549 \mathrm{e}-01$ & $1.07108 \mathrm{e}-06$ & $1.43328 \mathrm{e}-01$ & $3.56280 \mathrm{e}+03$ & 0.8044 & 851.5301 \\
\hline $\mathrm{Pu}^{4+}$ & $1.51398 \mathrm{e}-01$ & $5.91823 \mathrm{e}-07$ & $1.25510 \mathrm{e}-01$ & $9.68259 \mathrm{e}+03$ & 0.7044 & 2314.1953 \\
\hline $\mathrm{U}^{4+}$ & $2.01270 \mathrm{e}-01$ & $1.41288 \mathrm{e}-06$ & $1.38374 \mathrm{e}-01$ & $7.16790 \mathrm{e}+03$ & 0.7766 & 1713.1682 \\
\hline $\mathrm{Th}^{4+}$ & $1.84948 \mathrm{e}-01$ & $4.51805 \mathrm{e}-06$ & $1.70340 \mathrm{e}-01$ & $1.89274 \mathrm{e}+03$ & 0.9560 & 452.3759 \\
\hline
\end{tabular}


Table S13. Final Optimized LJ Parameters for TIP3P-FB Water Model in Different 12-

$6 \mathrm{LJ}$ Forms

\begin{tabular}{|c|c|c|c|c|c|c|}
\hline Ion & $\begin{array}{c}\mathrm{C}_{6} \\
\left(\mathrm{~kJ} \mathrm{~mol}^{-1} \mathrm{~nm}^{6}\right)\end{array}$ & $\begin{array}{c}\mathrm{C}_{12} \\
\left(\mathrm{~kJ} \mathrm{~mol}^{-1} \mathrm{~nm}^{12}\right)\end{array}$ & $\begin{array}{c}\text { sigma } \\
(\mathrm{nm})\end{array}$ & $\begin{array}{l}\text { epsilon } \\
(\mathrm{kJ} / \mathrm{mol})\end{array}$ & $\begin{array}{c}R_{\min } / 2 \\
(\AA)\end{array}$ & $\begin{array}{c}\text { epsilon } \\
(\mathrm{kcal} / \mathrm{mol})\end{array}$ \\
\hline $\mathrm{Al}^{3+}$ & $8.41996 \mathrm{e}-05$ & $1.95916 \mathrm{e}-13$ & $3.64021 \mathrm{e}-02$ & $9.04673 \mathrm{e}+03$ & 0.2043 & 2162.2206 \\
\hline $\mathrm{Cr}^{3+}$ & $7.57156 \mathrm{e}-04$ & $1.09805 \mathrm{e}-10$ & $7.24835 \mathrm{e}-02$ & $1.30524 \mathrm{e}+03$ & 0.4068 & 311.9608 \\
\hline $\mathrm{Fe}^{3+}$ & $1.40339 \mathrm{e}-03$ & $7.40300 \mathrm{e}-11$ & $6.12404 \mathrm{e}-02$ & $6.65107 \mathrm{e}+03$ & 0.3437 & 1589.6445 \\
\hline $\operatorname{In}^{3+}$ & $3.66637 \mathrm{e}-03$ & $1.50633 \mathrm{e}-09$ & $8.62212 \mathrm{e}-02$ & $2.23096 \mathrm{e}+03$ & 0.4839 & 533.2121 \\
\hline $\mathrm{Lu}^{3+}$ & $1.45519 \mathrm{e}-02$ & $1.73776 \mathrm{e}-07$ & $1.51186 \mathrm{e}-01$ & $3.04643 \mathrm{e}+02$ & 0.8485 & 72.8115 \\
\hline $\mathrm{Tl}^{3+}$ & $1.72981 \mathrm{e}-02$ & $2.25293 \mathrm{e}-08$ & $1.04502 \mathrm{e}-01$ & $3.32041 \mathrm{e}+03$ & 0.5865 & 793.5969 \\
\hline $\operatorname{Tm}^{3+}$ & $1.83442 \mathrm{e}-02$ & $2.25968 \mathrm{e}-07$ & $1.51970 \mathrm{e}-01$ & $3.72299 \mathrm{e}+02$ & 0.8529 & 88.9816 \\
\hline $\mathrm{Er}^{3+}$ & $1.74029 \mathrm{e}-02$ & $2.40984 \mathrm{e}-07$ & $1.54963 \mathrm{e}-01$ & $3.14190 \mathrm{e}+02$ & 0.8697 & 75.0931 \\
\hline $\mathrm{Y}^{3+}$ & $1.31892 \mathrm{e}-02$ & $2.39987 \mathrm{e}-07$ & $1.62179 \mathrm{e}-01$ & $1.81214 \mathrm{e}+02$ & 0.9102 & 43.3112 \\
\hline $\mathrm{Dy}^{3+}$ & $1.14920 \mathrm{e}-02$ & $2.55647 \mathrm{e}-07$ & $1.67703 \mathrm{e}-01$ & $1.29149 \mathrm{e}+02$ & 0.9412 & 30.8674 \\
\hline $\mathrm{Tb}^{3+}$ & $1.50787 \mathrm{e}-02$ & $3.94525 \mathrm{e}-07$ & $1.72300 \mathrm{e}-01$ & $1.44076 \mathrm{e}+02$ & 0.9670 & 34.4350 \\
\hline $\mathrm{Gd}^{3+}$ & $1.22973 \mathrm{e}-02$ & $3.90454 \mathrm{e}-07$ & $1.77948 \mathrm{e}-01$ & $9.68261 \mathrm{e}+01$ & 0.9987 & 23.1420 \\
\hline $\mathrm{Eu}^{3+}$ & $2.53450 \mathrm{e}-02$ & $8.40685 \mathrm{e}-07$ & $1.79249 \mathrm{e}-01$ & $1.91026 \mathrm{e}+02$ & 1.0060 & 45.6562 \\
\hline $\mathrm{Sm}^{3+}$ & $2.38924 \mathrm{e}-02$ & $9.76938 \mathrm{e}-07$ & $1.85610 \mathrm{e}-01$ & $1.46080 \mathrm{e}+02$ & 1.0417 & 34.9140 \\
\hline $\mathrm{Nd}^{3+}$ & $3.02802 \mathrm{e}-02$ & $1.55477 \mathrm{e}-06$ & $1.92790 \mathrm{e}-01$ & $1.47432 \mathrm{e}+02$ & 1.0820 & 35.2371 \\
\hline $\operatorname{Pr}^{3+}$ & $5.15928 \mathrm{e}-02$ & $3.10476 \mathrm{e}-06$ & $1.97958 \mathrm{e}-01$ & $2.14334 \mathrm{e}+02$ & 1.1110 & 51.2271 \\
\hline $\mathrm{Ce}^{3+}$ & $3.69959 \mathrm{e}-02$ & $2.92789 \mathrm{e}-06$ & $2.07205 \mathrm{e}-01$ & $1.16867 \mathrm{e}+02$ & 1.1629 & 27.9319 \\
\hline $\mathrm{La}^{3+}$ & $2.08590 \mathrm{e}-02$ & $2.64688 \mathrm{e}-06$ & $2.24168 \mathrm{e}-01$ & $4.10952 \mathrm{e}+01$ & 1.2581 & 9.8220 \\
\hline $\mathrm{Hf}^{4+}$ & $2.12168 \mathrm{e}-02$ & $7.42180 \mathrm{e}-09$ & $8.39405 \mathrm{e}-02$ & $1.51632 \mathrm{e}+04$ & 0.4711 & 3624.0986 \\
\hline $\mathrm{Zr}^{4+}$ & $2.02449 \mathrm{e}-02$ & $9.75165 \mathrm{e}-09$ & $8.85375 \mathrm{e}-02$ & $1.05073 \mathrm{e}+04$ & 0.4969 & 2511.3081 \\
\hline $\mathrm{Ce}^{4+}$ & $1.28582 \mathrm{e}-01$ & $1.10232 \mathrm{e}-06$ & $1.43061 \mathrm{e}-01$ & $3.74966 \mathrm{e}+03$ & 0.8029 & 896.1898 \\
\hline $\mathrm{Pu}^{4+}$ & $1.43811 \mathrm{e}-01$ & $5.19075 \mathrm{e}-07$ & $1.23853 \mathrm{e}-01$ & $9.96074 \mathrm{e}+03$ & 0.6951 & 2380.6743 \\
\hline $\mathrm{U}^{4+}$ & $1.82751 \mathrm{e}-01$ & $1.16752 \mathrm{e}-06$ & $1.36218 \mathrm{e}-01$ & $7.15141 \mathrm{e}+03$ & 0.7645 & 1709.2280 \\
\hline $\mathrm{Th}^{4+}$ & $1.63896 \mathrm{e}-01$ & $3.48403 \mathrm{e}-06$ & $1.66438 \mathrm{e}-01$ & $1.92749 \mathrm{e}+03$ & 0.9341 & 460.6807 \\
\hline
\end{tabular}


Table S14. Final Optimized LJ Parameters for a99SB-disp Water Model in Different

\section{2-6 LJ Forms}

\begin{tabular}{|c|c|c|c|c|c|c|}
\hline Ion & $\begin{array}{c}\mathrm{C}_{6} \\
\left(\mathrm{~kJ} \mathrm{~mol}{ }^{-1} \mathrm{~nm}^{6}\right)\end{array}$ & $\begin{array}{c}\mathrm{C}_{12} \\
\left(\mathrm{~kJ} \mathrm{~mol}^{-1} \mathrm{~nm}^{12}\right)\end{array}$ & $\begin{array}{l}\text { sigma } \\
(\mathrm{nm})\end{array}$ & $\begin{array}{l}\text { epsilon } \\
(\mathrm{kJ} / \mathrm{mol})\end{array}$ & $\begin{array}{c}R_{\min } / 2 \\
(\AA)\end{array}$ & $\begin{array}{c}\text { epsilon } \\
(\mathrm{kcal} / \mathrm{mol})\end{array}$ \\
\hline $\mathrm{Al}^{3+}$ & $2.09935 \mathrm{e}-05$ & $9.87004 \mathrm{e}-15$ & $2.78851 \mathrm{e}-02$ & $1.11633 \mathrm{e}+04$ & 0.1565 & 2668.0871 \\
\hline $\mathrm{Cr}^{3+}$ & $3.92910 \mathrm{e}-04$ & $1.72678 \mathrm{e}-11$ & $5.94051 \mathrm{e}-02$ & $2.23507 \mathrm{e}+03$ & 0.3334 & 534.1952 \\
\hline $\mathrm{Fe}^{3+}$ & $1.92350 \mathrm{e}-03$ & $1.03070 \mathrm{e}-10$ & $6.14007 \mathrm{e}-02$ & $8.97412 \mathrm{e}+03$ & 0.3446 & 2144.8651 \\
\hline $\operatorname{In}^{3+}$ & $3.68087 \mathrm{e}-03$ & $8.64069 \mathrm{e}-10$ & 7.85416e-02 & $3.92005 \mathrm{e}+03$ & 0.4408 & 936.9146 \\
\hline $\mathrm{Lu}^{3+}$ & $1.62129 \mathrm{e}-02$ & $9.65540 \mathrm{e}-08$ & $1.34633 \mathrm{e}-01$ & $6.80600 \mathrm{e}+02$ & 0.7556 & 162.6672 \\
\hline $\mathrm{Tl}^{3+}$ & $9.60334 \mathrm{e}-03$ & $5.83436 \mathrm{e}-09$ & $9.20298 \mathrm{e}-02$ & $3.95177 \mathrm{e}+03$ & 0.5165 & 944.4957 \\
\hline $\operatorname{Tm}^{3+}$ & $2.05355 \mathrm{e}-02$ & $1.37391 \mathrm{e}-07$ & $1.37270 \mathrm{e}-01$ & $7.67347 \mathrm{e}+02$ & 0.7704 & 183.4003 \\
\hline $\mathrm{Er}^{3+}$ & $1.96762 \mathrm{e}-02$ & $1.42013 \mathrm{e}-07$ & $1.39016 \mathrm{e}-01$ & $6.81541 \mathrm{e}+02$ & 0.7802 & 162.8921 \\
\hline $\mathrm{Y}^{3+}$ & $1.74795 \mathrm{e}-02$ & $1.49179 \mathrm{e}-07$ & $1.42954 \mathrm{e}-01$ & $5.12025 \mathrm{e}+02$ & 0.8023 & 122.3770 \\
\hline $\mathrm{Dy}^{3+}$ & $1.61180 \mathrm{e}-02$ & $1.56340 \mathrm{e}-07$ & $1.46036 \mathrm{e}-01$ & $4.15424 \mathrm{e}+02$ & 0.8196 & 99.2888 \\
\hline $\mathrm{Tb}^{3+}$ & $2.18433 \mathrm{e}-02$ & $2.73832 \mathrm{e}-07$ & $1.52415 \mathrm{e}-01$ & $4.35604 \mathrm{e}+02$ & 0.8554 & 104.1118 \\
\hline $\mathrm{Gd}^{3+}$ & $1.73187 \mathrm{e}-02$ & $2.36037 \mathrm{e}-07$ & $1.54553 \mathrm{e}-01$ & $3.17681 \mathrm{e}+02$ & 0.8674 & 75.9277 \\
\hline $\mathrm{Eu}^{3+}$ & $3.13083 \mathrm{e}-02$ & $5.71174 \mathrm{e}-07$ & $1.62250 \mathrm{e}-01$ & $4.29034 \mathrm{e}+02$ & 0.9106 & 102.5416 \\
\hline $\mathrm{Sm}^{3+}$ & $2.38661 \mathrm{e}-02$ & $4.63991 \mathrm{e}-07$ & $1.63979 \mathrm{e}-01$ & $3.06896 \mathrm{e}+02$ & 0.9203 & 73.3499 \\
\hline $\mathrm{Nd}^{3+}$ & $2.87047 \mathrm{e}-02$ & $7.69405 \mathrm{e}-07$ & $1.72995 \mathrm{e}-01$ & $2.67727 \mathrm{e}+02$ & 0.9709 & 63.9882 \\
\hline $\operatorname{Pr}^{3+}$ & $5.41385 \mathrm{e}-02$ & $2.02563 \mathrm{e}-06$ & $1.82884 \mathrm{e}-01$ & $3.61736 \mathrm{e}+02$ & 1.0264 & 86.4569 \\
\hline $\mathrm{Ce}^{3+}$ & $4.54636 \mathrm{e}-02$ & $2.03928 \mathrm{e}-06$ & $1.88496 \mathrm{e}-01$ & $2.53391 \mathrm{e}+02$ & 1.0579 & 60.5620 \\
\hline $\mathrm{La}^{3+}$ & $2.98525 \mathrm{e}-02$ & $1.66574 \mathrm{e}-06$ & $1.95481 \mathrm{e}-01$ & $1.33750 \mathrm{e}+02$ & 1.0971 & 31.9669 \\
\hline $\mathrm{Hf}^{4+}$ & $1.27763 \mathrm{e}-02$ & $2.35538 \mathrm{e}-09$ & $7.54413 \mathrm{e}-02$ & $1.73257 \mathrm{e}+04$ & 0.4234 & 4140.9501 \\
\hline $\mathrm{Zr}^{4+}$ & $1.86955 \mathrm{e}-02$ & $6.56484 \mathrm{e}-09$ & $8.39939 \mathrm{e}-02$ & $1.33104 \mathrm{e}+04$ & 0.4714 & 3181.2661 \\
\hline $\mathrm{Ce}^{4+}$ & $1.19055 \mathrm{e}-01$ & 7.19187e-07 & $1.34953 \mathrm{e}-01$ & $4.92710 \mathrm{e}+03$ & 0.7574 & 1177.6060 \\
\hline $\mathrm{Pu}^{4+}$ & $1.51766 \mathrm{e}-01$ & $5.00837 \mathrm{e}-07$ & $1.22017 \mathrm{e}-01$ & $1.14972 \mathrm{e}+04$ & 0.6848 & 2747.8942 \\
\hline $\mathrm{U}^{4+}$ & $1.45949 \mathrm{e}-01$ & $6.60463 \mathrm{e}-07$ & $1.28610 \mathrm{e}-01$ & $8.06291 \mathrm{e}+03$ & 0.7218 & 1927.0811 \\
\hline $\mathrm{Th}^{4+}$ & $1.86952 \mathrm{e}-01$ & $3.10176 \mathrm{e}-06$ & $1.59703 \mathrm{e}-01$ & $2.81703 \mathrm{e}+03$ & 0.8963 & 673.2867 \\
\hline
\end{tabular}


Table S15. Final Optimized LJ Parameters for TIP4P-Ew Water Model in Different 12-

\section{$6 \mathrm{LJ}$ Forms}

\begin{tabular}{|c|c|c|c|c|c|c|}
\hline Ion & $\begin{array}{c}\mathrm{C}_{6} \\
\left(\mathrm{~kJ} \mathrm{~mol}{ }^{-1} \mathrm{~nm}^{6}\right)\end{array}$ & $\begin{array}{c}\mathrm{C}_{12} \\
\left(\mathrm{~kJ} \mathrm{~mol}^{-1} \mathrm{~nm}^{12}\right)\end{array}$ & $\begin{array}{l}\text { sigma } \\
(\mathrm{nm})\end{array}$ & $\begin{array}{l}\text { epsilon } \\
(\mathrm{kJ} / \mathrm{mol})\end{array}$ & $\begin{array}{c}R_{\min } / 2 \\
(\AA)\end{array}$ & $\begin{array}{c}\text { epsilon } \\
(\mathrm{kcal} / \mathrm{mol})\end{array}$ \\
\hline $\mathrm{Al}^{3+}$ & $1.12447 \mathrm{e}-04$ & $1.84818 \mathrm{e}-13$ & $3.43531 \mathrm{e}-02$ & $1.71038 \mathrm{e}+04$ & 0.1928 & 4087.8975 \\
\hline $\mathrm{Cr}^{3+}$ & $6.16680 \mathrm{e}-04$ & $2.71999 \mathrm{e}-11$ & $5.94408 \mathrm{e}-02$ & $3.49536 \mathrm{e}+03$ & 0.3336 & 835.4106 \\
\hline $\mathrm{Fe}^{3+}$ & $1.07970 \mathrm{e}-03$ & $2.62639 \mathrm{e}-11$ & $5.38281 \mathrm{e}-02$ & $1.10966 \mathrm{e}+04$ & 0.3021 & 2652.1617 \\
\hline $\operatorname{In}^{3+}$ & $5.22025 \mathrm{e}-03$ & $1.45121 \mathrm{e}-09$ & $8.07867 \mathrm{e}-02$ & $4.69453 \mathrm{e}+03$ & 0.4534 & 1122.0185 \\
\hline $\mathrm{Lu}^{3+}$ & $1.90442 \mathrm{e}-02$ & $1.06309 \mathrm{e}-07$ & $1.33189 \mathrm{e}-01$ & $8.52887 \mathrm{e}+02$ & 0.7475 & 203.8449 \\
\hline $\mathrm{Tl}^{3+}$ & $1.91386 \mathrm{e}-02$ & $1.67868 \mathrm{e}-08$ & $9.78385 \mathrm{e}-02$ & $5.45496 \mathrm{e}+03$ & 0.5491 & 1303.7671 \\
\hline $\operatorname{Tm}^{3+}$ & $3.15756 \mathrm{e}-02$ & $2.31073 \mathrm{e}-07$ & $1.39337 \mathrm{e}-01$ & $1.07868 \mathrm{e}+03$ & 0.7820 & 257.8101 \\
\hline $\mathrm{Er}^{3+}$ & $2.99901 \mathrm{e}-02$ & $2.34532 \mathrm{e}-07$ & $1.40887 \mathrm{e}-01$ & $9.58720 \mathrm{e}+02$ & 0.7907 & 229.1395 \\
\hline $\mathrm{Y}^{3+}$ & $2.27658 \mathrm{e}-02$ & $1.98250 \mathrm{e}-07$ & $1.43435 \mathrm{e}-01$ & $6.53569 \mathrm{e}+02$ & 0.8050 & 156.2068 \\
\hline $\mathrm{Dy}^{3+}$ & $2.02924 \mathrm{e}-02$ & $1.95396 \mathrm{e}-07$ & $1.45858 \mathrm{e}-01$ & $5.26856 \mathrm{e}+02$ & 0.8186 & 125.9215 \\
\hline $\mathrm{Tb}^{3+}$ & $2.73253 \mathrm{e}-02$ & $3.39921 \mathrm{e}-07$ & $1.52219 \mathrm{e}-01$ & $5.49151 \mathrm{e}+02$ & 0.8543 & 131.2502 \\
\hline $\mathrm{Gd}^{3+}$ & $2.30954 \mathrm{e}-02$ & $3.17613 \mathrm{e}-07$ & $1.54785 \mathrm{e}-01$ & $4.19848 \mathrm{e}+02$ & 0.8687 & 100.3460 \\
\hline $\mathrm{Eu}^{3+}$ & $4.30031 \mathrm{e}-02$ & 7.64096e-07 & $1.61538 \mathrm{e}-01$ & $6.05050 \mathrm{e}+02$ & 0.9066 & 144.6105 \\
\hline $\mathrm{Sm}^{3+}$ & $4.05938 \mathrm{e}-02$ & $8.46970 \mathrm{e}-07$ & $1.65921 \mathrm{e}-01$ & $4.86398 \mathrm{e}+02$ & 0.9312 & 116.2519 \\
\hline $\mathrm{Nd}^{3+}$ & $4.52033 \mathrm{e}-02$ & $1.19671 \mathrm{e}-06$ & $1.72638 \mathrm{e}-01$ & $4.26866 \mathrm{e}+02$ & 0.9689 & 102.0234 \\
\hline $\operatorname{Pr}^{3+}$ & $7.09349 \mathrm{e}-02$ & $2.52637 \mathrm{e}-06$ & $1.81387 \mathrm{e}-01$ & $4.97924 \mathrm{e}+02$ & 1.0180 & 119.0066 \\
\hline $\mathrm{Ce}^{3+}$ & $7.46454 \mathrm{e}-02$ & $3.27306 \mathrm{e}-06$ & $1.87784 \mathrm{e}-01$ & $4.25590 \mathrm{e}+02$ & 1.0539 & 101.7185 \\
\hline $\mathrm{La}^{3+}$ & $3.91466 \mathrm{e}-02$ & $2.04709 \mathrm{e}-06$ & $1.93378 \mathrm{e}-01$ & $1.87151 \mathrm{e}+02$ & 1.0853 & 44.7301 \\
\hline $\mathrm{Hf}^{4+}$ & $2.13050 \mathrm{e}-02$ & $4.88665 \mathrm{e}-09$ & $7.82387 \mathrm{e}-02$ & $2.32216 \mathrm{e}+04$ & 0.4391 & 5550.0897 \\
\hline $\mathrm{Zr}^{4+}$ & $2.63749 \mathrm{e}-02$ & $9.82950 \mathrm{e}-09$ & $8.48314 \mathrm{e}-02$ & $1.76926 \mathrm{e}+04$ & 0.4761 & 4228.6331 \\
\hline $\mathrm{Ce}^{4+}$ & $1.53423 \mathrm{e}-01$ & $9.14383 \mathrm{e}-07$ & $1.34650 \mathrm{e}-01$ & $6.43564 \mathrm{e}+03$ & 0.7557 & 1538.1546 \\
\hline $\mathrm{Pu}^{4+}$ & $2.14097 \mathrm{e}-01$ & $7.50359 \mathrm{e}-07$ & $1.23247 \mathrm{e}-01$ & $1.52719 \mathrm{e}+04$ & 0.6917 & 3650.0600 \\
\hline $\mathrm{U}^{4+}$ & $2.22387 \mathrm{e}-01$ & $1.11832 \mathrm{e}-06$ & $1.30891 \mathrm{e}-01$ & $1.10558 \mathrm{e}+04$ & 0.7346 & 2642.4088 \\
\hline $\mathrm{Th}^{4+}$ & $1.86126 \mathrm{e}-01$ & $2.52443 \mathrm{e}-06$ & $1.54428 \mathrm{e}-01$ & $3.43077 \mathrm{e}+03$ & 0.8667 & 819.9739 \\
\hline
\end{tabular}


Table S16. Final Optimized LJ Parameters for OPC Water Model in Different 12-6 LJ

\section{Forms}

\begin{tabular}{|c|c|c|c|c|c|c|}
\hline Ion & $\begin{array}{c}\mathrm{C}_{6} \\
\left(\mathrm{~kJ} \mathrm{~mol}^{-1} \mathrm{~nm}^{6}\right)\end{array}$ & $\begin{array}{c}\mathrm{C}_{12} \\
\left(\mathrm{~kJ} \mathrm{~mol}^{-1} \mathrm{~nm}^{12}\right)\end{array}$ & $\begin{array}{l}\text { sigma } \\
(\mathrm{nm})\end{array}$ & $\begin{array}{l}\text { epsilon } \\
(\mathrm{kJ} / \mathrm{mol})\end{array}$ & $\begin{array}{c}R_{\min } / 2 \\
(\AA)\end{array}$ & $\begin{array}{c}\text { epsilon } \\
(\mathrm{kcal} / \mathrm{mol})\end{array}$ \\
\hline $\mathrm{Al}^{3+}$ & $3.19118 \mathrm{e}-05$ & $2.99400 \mathrm{e}-14$ & $3.12884 \mathrm{e}-02$ & $8.50338 \mathrm{e}+03$ & 0.1756 & 2032.3570 \\
\hline $\mathrm{Cr}^{3+}$ & $5.96535 \mathrm{e}-04$ & $5.84816 \mathrm{e}-11$ & $6.79043 \mathrm{e}-02$ & $1.52122 \mathrm{e}+03$ & 0.3811 & 363.5800 \\
\hline $\mathrm{Fe}^{3+}$ & $1.82026 \mathrm{e}-03$ & $1.19576 \mathrm{e}-10$ & $6.35211 \mathrm{e}-02$ & $6.92734 \mathrm{e}+03$ & 0.3565 & 1655.6733 \\
\hline $\operatorname{In}^{3+}$ & $3.73070 \mathrm{e}-03$ & $1.28685 \mathrm{e}-09$ & $8.37445 \mathrm{e}-02$ & $2.70391 \mathrm{e}+03$ & 0.4700 & 646.2492 \\
\hline $\mathrm{Lu}^{3+}$ & $1.50310 \mathrm{e}-02$ & $1.38588 \mathrm{e}-07$ & $1.44807 \mathrm{e}-01$ & $4.07560 \mathrm{e}+02$ & 0.8127 & 97.4092 \\
\hline $\mathrm{Tl}^{3+}$ & $1.22510 \mathrm{e}-02$ & $1.18085 \mathrm{e}-08$ & $9.93887 \mathrm{e}-02$ & $3.17754 \mathrm{e}+03$ & 0.5578 & 759.4514 \\
\hline $\operatorname{Tm}^{3+}$ & $1.85719 \mathrm{e}-02$ & $1.83469 \mathrm{e}-07$ & $1.46482 \mathrm{e}-01$ & $4.69993 \mathrm{e}+02$ & 0.8221 & 112.3311 \\
\hline $\mathrm{Er}^{3+}$ & $1.77101 \mathrm{e}-02$ & $1.93194 \mathrm{e}-07$ & $1.48923 \mathrm{e}-01$ & $4.05874 \mathrm{e}+02$ & 0.8358 & 97.0063 \\
\hline $\mathrm{Y}^{3+}$ & $1.50704 \mathrm{e}-02$ & $2.07252 \mathrm{e}-07$ & $1.54785 \mathrm{e}-01$ & $2.73963 \mathrm{e}+02$ & 0.8687 & 65.4787 \\
\hline $\mathrm{Dy}^{3+}$ & $1.49744 \mathrm{e}-02$ & $2.37820 \mathrm{e}-07$ & $1.58544 \mathrm{e}-01$ & $2.35717 \mathrm{e}+02$ & 0.8898 & 56.3378 \\
\hline $\mathrm{Tb}^{3+}$ & $1.93268 \mathrm{e}-02$ & $3.71596 \mathrm{e}-07$ & $1.63676 \mathrm{e}-01$ & $2.51299 \mathrm{e}+02$ & 0.9186 & 60.0620 \\
\hline $\mathrm{Gd}^{3+}$ & $1.40074 \mathrm{e}-02$ & $2.93198 \mathrm{e}-07$ & $1.66010 \mathrm{e}-01$ & $1.67298 \mathrm{e}+02$ & 0.9317 & 39.9852 \\
\hline $\mathrm{Eu}^{3+}$ & $2.70118 \mathrm{e}-02$ & $6.88104 \mathrm{e}-07$ & $1.71534 \mathrm{e}-01$ & $2.65089 \mathrm{e}+02$ & 0.9627 & 63.3578 \\
\hline $\mathrm{Sm}^{3+}$ & $1.96000 \mathrm{e}-02$ & $6.01672 \mathrm{e}-07$ & $1.76950 \mathrm{e}-01$ & $1.59622 \mathrm{e}+02$ & 0.9931 & 38.1505 \\
\hline $\mathrm{Nd}^{3+}$ & $2.24804 \mathrm{e}-02$ & $9.15498 \mathrm{e}-07$ & $1.85485 \mathrm{e}-01$ & $1.38004 \mathrm{e}+02$ & 1.0410 & 32.9837 \\
\hline $\operatorname{Pr}^{3+}$ & $4.75776 \mathrm{e}-02$ & $2.44429 \mathrm{e}-06$ & $1.92808 \mathrm{e}-01$ & $2.31522 \mathrm{e}+02$ & 1.0821 & 55.3350 \\
\hline $\mathrm{Ce}^{3+}$ & $4.14005 \mathrm{e}-02$ & $2.62761 \mathrm{e}-06$ & $1.99722 \mathrm{e}-01$ & $1.63076 \mathrm{e}+02$ & 1.1209 & 38.9762 \\
\hline $\mathrm{La}^{3+}$ & $2.08220 \mathrm{e}-02$ & $1.99753 \mathrm{e}-06$ & $2.13958 \mathrm{e}-01$ & $5.42615 \mathrm{e}+01$ & 1.2008 & 12.9688 \\
\hline $\mathrm{Hf}^{4+}$ & $1.25863 \mathrm{e}-02$ & $2.79350 \mathrm{e}-09$ & 7.78111e-02 & $1.41772 \mathrm{e}+04$ & 0.4367 & 3388.4415 \\
\hline $\mathrm{Zr}^{4+}$ & $2.15209 \mathrm{e}-02$ & $1.06065 \mathrm{e}-08$ & $8.88761 \mathrm{e}-02$ & $1.09167 \mathrm{e}+04$ & 0.4988 & 2609.1557 \\
\hline $\mathrm{Ce}^{4+}$ & $1.11415 \mathrm{e}-01$ & $8.17206 \mathrm{e}-07$ & $1.39390 \mathrm{e}-01$ & $3.79745 \mathrm{e}+03$ & 0.7823 & 907.6115 \\
\hline $\mathrm{Pu}^{4+}$ & $1.48016 \mathrm{e}-01$ & $5.62041 \mathrm{e}-07$ & $1.24904 \mathrm{e}-01$ & $9.74522 \mathrm{e}+03$ & 0.7010 & 2329.1636 \\
\hline $\mathrm{U}^{4+}$ & $1.44440 \mathrm{e}-01$ & $7.77009 \mathrm{e}-07$ & $1.32370 \mathrm{e}-01$ & $6.71261 \mathrm{e}+03$ & 0.7429 & 1604.3533 \\
\hline $\mathrm{Th}^{4+}$ & $1.55321 \mathrm{e}-01$ & $2.97858 \mathrm{e}-06$ & $1.63605 \mathrm{e}-01$ & $2.02484 \mathrm{e}+03$ & 0.9182 & 483.9494 \\
\hline
\end{tabular}


Table S17. Final Optimized LJ Parameters for TIP4P/2005 Water Model in Different

\section{2-6 LJ Forms}

\begin{tabular}{|c|c|c|c|c|c|c|}
\hline Ion & $\begin{array}{c}\mathrm{C}_{6} \\
\left(\mathrm{~kJ} \mathrm{~mol}^{-1} \mathrm{~nm}^{6}\right)\end{array}$ & $\begin{array}{c}\mathrm{C}_{12} \\
\left(\mathrm{~kJ} \mathrm{~mol}^{-1} \mathrm{~nm}^{12}\right)\end{array}$ & $\begin{array}{l}\text { sigma } \\
(\mathrm{nm})\end{array}$ & $\begin{array}{l}\text { epsilon } \\
(\mathrm{kJ} / \mathrm{mol})\end{array}$ & $\begin{array}{c}R_{\min } / 2 \\
(\AA)\end{array}$ & $\begin{array}{c}\text { epsilon } \\
(\mathrm{kcal} / \mathrm{mol})\end{array}$ \\
\hline $\mathrm{Al}^{3+}$ & $7.32554 \mathrm{e}-05$ & $7.92002 \mathrm{e}-14$ & $3.20367 \mathrm{e}-02$ & $1.69392 \mathrm{e}+04$ & 0.1798 & 4048.5546 \\
\hline $\mathrm{Cr}^{3+}$ & $5.90793 \mathrm{e}-04$ & $2.15883 \mathrm{e}-11$ & $5.76055 \mathrm{e}-02$ & $4.04196 \mathrm{e}+03$ & 0.3233 & 966.0509 \\
\hline $\mathrm{Fe}^{3+}$ & $1.47143 \mathrm{e}-03$ & $4.53031 \mathrm{e}-11$ & $5.59841 \mathrm{e}-02$ & $1.19479 \mathrm{e}+04$ & 0.3142 & 2855.6172 \\
\hline $\operatorname{In}^{3+}$ & $4.76755 \mathrm{e}-03$ & $1.09053 \mathrm{e}-09$ & 7.82031e-02 & $5.21065 \mathrm{e}+03$ & 0.4389 & 1245.3746 \\
\hline $\mathrm{Lu}^{3+}$ & $2.22944 \mathrm{e}-02$ & $1.20514 \mathrm{e}-07$ & $1.32477 \mathrm{e}-01$ & $1.03108 \mathrm{e}+03$ & 0.7435 & 246.4336 \\
\hline $\mathrm{Tl}^{3+}$ & $1.81041 \mathrm{e}-02$ & $1.44445 \mathrm{e}-08$ & $9.63062 \mathrm{e}-02$ & $5.67272 \mathrm{e}+03$ & 0.5405 & 1355.8137 \\
\hline $\operatorname{Tm}^{3+}$ & $2.74573 \mathrm{e}-02$ & $1.66788 \mathrm{e}-07$ & $1.35078 \mathrm{e}-01$ & $1.13003 \mathrm{e}+03$ & 0.7581 & 270.0847 \\
\hline $\mathrm{Er}^{3+}$ & $2.72399 \mathrm{e}-02$ & $1.80825 \mathrm{e}-07$ & $1.37091 \mathrm{e}-01$ & $1.02587 \mathrm{e}+03$ & 0.7694 & 245.1884 \\
\hline $\mathrm{Y}^{3+}$ & $2.02743 \mathrm{e}-02$ & $1.40682 \mathrm{e}-07$ & $1.38107 \mathrm{e}-01$ & $7.30452 \mathrm{e}+02$ & 0.7751 & 174.5823 \\
\hline $\mathrm{Dy}^{3+}$ & $2.26678 \mathrm{e}-02$ & $1.95194 \mathrm{e}-07$ & $1.43167 \mathrm{e}-01$ & $6.58100 \mathrm{e}+02$ & 0.8035 & 157.2896 \\
\hline $\mathrm{Tb}^{3+}$ & $2.83831 \mathrm{e}-02$ & $3.06738 \mathrm{e}-07$ & $1.48691 \mathrm{e}-01$ & $6.56586 \mathrm{e}+02$ & 0.8345 & 156.9278 \\
\hline $\mathrm{Gd}^{3+}$ & $2.22492 \mathrm{e}-02$ & $2.49044 \mathrm{e}-07$ & $1.49564 \mathrm{e}-01$ & $4.96926 \mathrm{e}+02$ & 0.8394 & 118.7682 \\
\hline $\mathrm{Eu}^{3+}$ & $4.01675 \mathrm{e}-02$ & $6.08411 \mathrm{e}-07$ & $1.57297 \mathrm{e}-01$ & $6.62967 \mathrm{e}+02$ & 0.8828 & 158.4528 \\
\hline $\mathrm{Sm}^{3+}$ & $3.57861 \mathrm{e}-02$ & $6.17983 \mathrm{e}-07$ & $1.60772 \mathrm{e}-01$ & $5.18074 \mathrm{e}+02$ & 0.9023 & 123.8226 \\
\hline $\mathrm{Nd}^{3+}$ & $4.25888 \mathrm{e}-02$ & $9.68144 \mathrm{e}-07$ & $1.68309 \mathrm{e}-01$ & $4.68373 \mathrm{e}+02$ & 0.9446 & 111.9438 \\
\hline $\operatorname{Pr}^{3+}$ & $7.60232 \mathrm{e}-02$ & $2.41236 \mathrm{e}-06$ & $1.77930 \mathrm{e}-01$ & $5.98951 \mathrm{e}+02$ & 0.9986 & 143.1528 \\
\hline $\mathrm{Ce}^{3+}$ & $7.19003 \mathrm{e}-02$ & $2.81845 \mathrm{e}-06$ & $1.84309 \mathrm{e}-01$ & $4.58555 \mathrm{e}+02$ & 1.0344 & 109.5973 \\
\hline $\mathrm{La}^{3+}$ & $4.79254 \mathrm{e}-02$ & $2.23398 \mathrm{e}-06$ & $1.89708 \mathrm{e}-01$ & $2.57035 \mathrm{e}+02$ & 1.0647 & 61.4328 \\
\hline $\mathrm{Hf}^{4+}$ & $2.11457 \mathrm{e}-02$ & $4.64827 \mathrm{e}-09$ & $7.76864 \mathrm{e}-02$ & $2.40487 \mathrm{e}+04$ & 0.4360 & 5747.7782 \\
\hline $\mathrm{Zr}^{4+}$ & $2.47883 \mathrm{e}-02$ & $8.36649 \mathrm{e}-09$ & $8.34416 \mathrm{e}-02$ & $1.83608 \mathrm{e}+04$ & 0.4683 & 4388.3373 \\
\hline $\mathrm{Ce}^{4+}$ & $1.58138 \mathrm{e}-01$ & $9.20277 \mathrm{e}-07$ & $1.34116 \mathrm{e}-01$ & $6.79347 \mathrm{e}+03$ & 0.7527 & 1623.6783 \\
\hline $\mathrm{Pu}^{4+}$ & $2.15194 \mathrm{e}-01$ & $7.56152 \mathrm{e}-07$ & $1.23300 \mathrm{e}-01$ & $1.53106 \mathrm{e}+04$ & 0.6920 & 3659.3167 \\
\hline $\mathrm{U}^{4+}$ & $1.87816 \mathrm{e}-01$ & $8.05031 \mathrm{e}-07$ & $1.27452 \mathrm{e}-01$ & $1.09545 \mathrm{e}+04$ & 0.7153 & 2618.1830 \\
\hline $\mathrm{Th}^{4+}$ & $1.99680 \mathrm{e}-01$ & $2.61769 \mathrm{e}-06$ & $1.53555 \mathrm{e}-01$ & $3.80795 \mathrm{e}+03$ & 0.8618 & 910.1228 \\
\hline
\end{tabular}


Table S18. Final Optimized LJ Parameters for TIP4P-D Water Model in Different 12-

\section{$6 \mathrm{LJ}$ Forms}

\begin{tabular}{|c|c|c|c|c|c|c|}
\hline Ja & $\mathrm{C}_{6}$ & $\mathrm{C}_{12}$ & sigma & epsilon & $R_{\min } / 2$ & epsilon \\
\hline 1011 & $\left(\mathrm{~kJ} \mathrm{~mol}^{-1} \mathrm{~nm}^{6}\right)$ & $\left(\mathrm{kJ} \mathrm{mol}^{-1} \mathrm{~nm}^{12}\right)$ & $(\mathrm{nm})$ & $(\mathrm{kJ} / \mathrm{mol})$ & $(\AA)$ & $(\mathrm{kcal} / \mathrm{mol})$ \\
\hline $\mathrm{Al}^{3+}$ & $2.65000 \mathrm{e}-05$ & $1.44958 \mathrm{e}-14$ & $2.85978 \mathrm{e}-02$ & $1.21113 \mathrm{e}+04$ & 0.1605 & 2894.6762 \\
\hline $\mathrm{Cr}^{3+}$ & $4.19704 \mathrm{e}-04$ & $1.74716 \mathrm{e}-11$ & $5.88706 \mathrm{e}-02$ & $2.52053 \mathrm{e}+03$ & 0.3304 & 602.4208 \\
\hline $\mathrm{Fe}^{3+}$ & $1.67463 \mathrm{e}-03$ & $7.41289 \mathrm{e}-11$ & $5.94764 \mathrm{e}-02$ & $9.45783 \mathrm{e}+03$ & 0.3338 & 2260.4765 \\
\hline $\operatorname{In}^{3+}$ & $3.71588 \mathrm{e}-03$ & $8.36126 \mathrm{e}-10$ & $7.79893 \mathrm{e}-02$ & $4.12849 \mathrm{e}+03$ & 0.4377 & 986.7338 \\
\hline $\mathrm{Lu}^{3+}$ & $1.70967 \mathrm{e}-02$ & $9.99712 \mathrm{e}-08$ & $1.34223 \mathrm{e}-01$ & $7.30957 \mathrm{e}+02$ & 0.7533 & 174.7029 \\
\hline $\mathrm{Tl}^{3+}$ & $1.01295 \mathrm{e}-02$ & $6.10417 \mathrm{e}-09$ & $9.19051 \mathrm{e}-02$ & $4.20234 \mathrm{e}+03$ & 0.5158 & 1004.3845 \\
\hline $\operatorname{Tm}^{3+}$ & $2.13545 \mathrm{e}-02$ & $1.38908 \mathrm{e}-07$ & $1.36628 \mathrm{e}-01$ & $8.20714 \mathrm{e}+02$ & 0.7668 & 196.1553 \\
\hline $\mathrm{Er}^{3+}$ & $2.05834 \mathrm{e}-02$ & $1.44605 \mathrm{e}-07$ & $1.38392 \mathrm{e}-01$ & $7.32473 \mathrm{e}+02$ & 0.7767 & 175.0653 \\
\hline $\mathrm{Y}^{3+}$ & $1.81338 \mathrm{e}-02$ & $1.50983 \mathrm{e}-07$ & $1.42366 \mathrm{e}-01$ & $5.44492 \mathrm{e}+02$ & 0.7990 & 130.1368 \\
\hline $\mathrm{Dy}^{3+}$ & $1.74766 \mathrm{e}-02$ & $1.64613 \mathrm{e}-07$ & $1.45323 \mathrm{e}-01$ & $4.63865 \mathrm{e}+02$ & 0.8156 & 110.8664 \\
\hline $\mathrm{Tb}^{3+}$ & $2.27718 \mathrm{e}-02$ & $2.77169 \mathrm{e}-07$ & $1.51667 \mathrm{e}-01$ & $4.67726 \mathrm{e}+02$ & 0.8512 & 111.7892 \\
\hline $\mathrm{Gd}^{3+}$ & $1.70665 \mathrm{e}-02$ & $2.18795 \mathrm{e}-07$ & $1.52985 \mathrm{e}-01$ & $3.32806 \mathrm{e}+02$ & 0.8586 & 79.5426 \\
\hline $\mathrm{Eu}^{3+}$ & $3.28893 \mathrm{e}-02$ & $5.79373 \mathrm{e}-07$ & $1.61306 \mathrm{e}-01$ & $4.66758 \mathrm{e}+02$ & 0.9053 & 111.5578 \\
\hline $\mathrm{Sm}^{3+}$ & $2.73843 \mathrm{e}-02$ & $5.46075 \mathrm{e}-07$ & $1.64674 \mathrm{e}-01$ & $3.43314 \mathrm{e}+02$ & 0.9242 & 82.0540 \\
\hline $\mathrm{Nd}^{3+}$ & $3.10133 \mathrm{e}-02$ & $8.03427 \mathrm{e}-07$ & $1.72015 \mathrm{e}-01$ & $2.99289 \mathrm{e}+02$ & 0.9654 & 71.5319 \\
\hline $\operatorname{Pr}^{3+}$ & $6.04699 \mathrm{e}-02$ & $2.20103 \mathrm{e}-06$ & $1.82046 \mathrm{e}-01$ & $4.15329 \mathrm{e}+02$ & 1.0217 & 99.2659 \\
\hline $\mathrm{Ce}^{3+}$ & $5.18747 \mathrm{e}-02$ & $2.29540 \mathrm{e}-06$ & $1.88069 \mathrm{e}-01$ & $2.93084 \mathrm{e}+02$ & 1.0555 & 70.0487 \\
\hline $\mathrm{La}^{3+}$ & $3.04753 \mathrm{e}-02$ & $1.66819 \mathrm{e}-06$ & $1.94857 \mathrm{e}-01$ & $1.39185 \mathrm{e}+02$ & 1.0936 & 33.2660 \\
\hline $\mathrm{Hf}^{4+}$ & $1.32848 \mathrm{e}-02$ & $2.36024 \mathrm{e}-09$ & 7.49780e-02 & $1.86935 \mathrm{e}+04$ & 0.4208 & 4467.8646 \\
\hline $\mathrm{Zr}^{4+}$ & $2.22828 \mathrm{e}-02$ & $8.50523 \mathrm{e}-09$ & $8.51699 \mathrm{e}-02$ & $1.45946 \mathrm{e}+04$ & 0.4780 & 3488.1889 \\
\hline $\mathrm{Ce}^{4+}$ & $1.17533 \mathrm{e}-01$ & $6.60335 \mathrm{e}-07$ & $1.33332 \mathrm{e}-01$ & $5.22988 \mathrm{e}+03$ & 0.7483 & 1249.9712 \\
\hline $\mathrm{Pu}^{4+}$ & $1.58264 \mathrm{e}-01$ & $5.10049 \mathrm{e}-07$ & $1.21536 \mathrm{e}-01$ & $1.22770 \mathrm{e}+04$ & 0.6821 & 2934.2695 \\
\hline $\mathrm{U}^{4+}$ & $1.49750 \mathrm{e}-01$ & $6.47269 \mathrm{e}-07$ & $1.27630 \mathrm{e}-01$ & $8.66147 \mathrm{e}+03$ & 0.7163 & 2070.1413 \\
\hline $\mathrm{Th}^{4+}$ & $2.05824 \mathrm{e}-01$ & $3.44462 \mathrm{e}-06$ & $1.59934 \mathrm{e}-01$ & $3.07462 \mathrm{e}+03$ & 0.8976 & 734.8522 \\
\hline
\end{tabular}


Table S19. Final Optimized LJ Parameters for TIP4P-FB Water Model in Different 12-

\section{$6 \mathrm{LJ}$ Forms}

\begin{tabular}{|c|c|c|c|c|c|c|}
\hline Ja & $\mathrm{C}_{6}$ & $\mathrm{C}_{12}$ & sigma & epsilon & $R_{\min } / 2$ & epsilon \\
\hline 1011 & $\left(\mathrm{~kJ} \mathrm{~mol}^{-1} \mathrm{~nm}^{6}\right)$ & $\left(\mathrm{kJ} \mathrm{mol}^{-1} \mathrm{~nm}^{12}\right)$ & $(\mathrm{nm})$ & $(\mathrm{kJ} / \mathrm{mol})$ & $(\AA)$ & (kcal/mol) \\
\hline $\mathrm{Al}^{3+}$ & $9.76554 \mathrm{e}-05$ & $1.92904 \mathrm{e}-13$ & $3.54221 \mathrm{e}-02$ & $1.23592 \mathrm{e}+04$ & 0.1988 & 2953.9286 \\
\hline $\mathrm{Cr}^{3+}$ & $6.07739 \mathrm{e}-04$ & $4.20517 \mathrm{e}-11$ & $6.40734 \mathrm{e}-02$ & $2.19579 \mathrm{e}+03$ & 0.3596 & 524.8074 \\
\hline $\mathrm{Fe}^{3+}$ & $1.54703 \mathrm{e}-03$ & $6.92223 \mathrm{e}-11$ & $5.95833 \mathrm{e}-02$ & $8.64354 \mathrm{e}+03$ & 0.3344 & 2065.8558 \\
\hline $\operatorname{In}^{3+}$ & $4.30489 \mathrm{e}-03$ & $1.36764 \mathrm{e}-09$ & $8.26041 \mathrm{e}-02$ & $3.38760 \mathrm{e}+03$ & 0.4636 & 809.6550 \\
\hline $\mathrm{Lu}^{3+}$ & $1.56196 \mathrm{e}-02$ & $1.18220 \mathrm{e}-07$ & $1.40121 \mathrm{e}-01$ & $5.15931 \mathrm{e}+02$ & 0.7864 & 123.3105 \\
\hline $\mathrm{Tl}^{3+}$ & $1.56006 \mathrm{e}-02$ & $1.51506 \mathrm{e}-08$ & $9.95134 \mathrm{e}-02$ & $4.01598 \mathrm{e}+03$ & 0.5585 & 959.8425 \\
\hline $\operatorname{Tm}^{3+}$ & $1.96988 \mathrm{e}-02$ & $1.62416 \mathrm{e}-07$ & $1.42134 \mathrm{e}-01$ & $5.97299 \mathrm{e}+02$ & 0.7977 & 142.7579 \\
\hline $\mathrm{Er}^{3+}$ & $1.85642 \mathrm{e}-02$ & $1.68151 \mathrm{e}-07$ & $1.44379 \mathrm{e}-01$ & $5.12379 \mathrm{e}+02$ & 0.8103 & 122.4616 \\
\hline $\mathrm{Y}^{3+}$ & $1.65135 \mathrm{e}-02$ & $1.89245 \mathrm{e}-07$ & $1.50152 \mathrm{e}-01$ & $3.60240 \mathrm{e}+02$ & 0.8427 & 86.0994 \\
\hline $\mathrm{Dy}^{3+}$ & $1.60172 \mathrm{e}-02$ & $2.13513 \mathrm{e}-07$ & $1.53983 \mathrm{e}-01$ & $3.00394 \mathrm{e}+02$ & 0.8642 & 71.7960 \\
\hline $\mathrm{Tb}^{3+}$ & $2.22657 \mathrm{e}-02$ & $3.69165 \mathrm{e}-07$ & $1.59685 \mathrm{e}-01$ & $3.35731 \mathrm{e}+02$ & 0.8962 & 80.2417 \\
\hline $\mathrm{Gd}^{3+}$ & $1.73424 \mathrm{e}-02$ & $3.14100 \mathrm{e}-07$ & $1.62054 \mathrm{e}-01$ & $2.39382 \mathrm{e}+02$ & 0.9095 & 57.2137 \\
\hline $\mathrm{Eu}^{3+}$ & $4.02275 \mathrm{e}-02$ & $9.25540 \mathrm{e}-07$ & $1.68647 \mathrm{e}-01$ & $4.37111 \mathrm{e}+02$ & 0.9465 & 104.4720 \\
\hline $\mathrm{Sm}^{3+}$ & $3.35346 \mathrm{e}-02$ & $8.78484 \mathrm{e}-07$ & $1.72335 \mathrm{e}-01$ & $3.20031 \mathrm{e}+02$ & 0.9672 & 76.4892 \\
\hline $\mathrm{Nd}^{3+}$ & $3.36819 \mathrm{e}-02$ & $1.13669 \mathrm{e}-06$ & $1.79766 \mathrm{e}-01$ & $2.49512 \mathrm{e}+02$ & 1.0089 & 59.6348 \\
\hline $\operatorname{Pr}^{3+}$ & $5.87270 \mathrm{e}-02$ & $2.58240 \mathrm{e}-06$ & $1.87873 \mathrm{e}-01$ & $3.33881 \mathrm{e}+02$ & 1.0544 & 79.7995 \\
\hline $\mathrm{Ce}^{3+}$ & $5.21794 \mathrm{e}-02$ & $2.85000 \mathrm{e}-06$ & $1.94786 \mathrm{e}-01$ & $2.38832 \mathrm{e}+02$ & 1.0932 & 57.0821 \\
\hline $\mathrm{La}^{3+}$ & $3.07305 \mathrm{e}-02$ & $2.24278 \mathrm{e}-06$ & $2.04426 \mathrm{e}-01$ & $1.05267 \mathrm{e}+02$ & 1.1473 & 25.1594 \\
\hline $\mathrm{Hf}^{4+}$ & $1.68195 \mathrm{e}-02$ & $3.96987 \mathrm{e}-09$ & $7.86129 \mathrm{e}-02$ & $1.78152 \mathrm{e}+04$ & 0.4412 & 4257.9445 \\
\hline $\mathrm{Zr}^{4+}$ & $2.28897 \mathrm{e}-02$ & $9.75950 \mathrm{e}-09$ & $8.67557 \mathrm{e}-02$ & $1.34212 \mathrm{e}+04$ & 0.4869 & 3207.7462 \\
\hline $\mathrm{Ce}^{4+}$ & $1.43641 \mathrm{e}-01$ & $1.05926 \mathrm{e}-06$ & $1.39515 \mathrm{e}-01$ & $4.86958 \mathrm{e}+03$ & 0.7830 & 1163.8580 \\
\hline $\mathrm{Pu}^{4+}$ & $1.71500 \mathrm{e}-01$ & $6.13163 \mathrm{e}-07$ & $1.23657 \mathrm{e}-01$ & $1.19920 \mathrm{e}+04$ & 0.6940 & 2866.1571 \\
\hline $\mathrm{U}^{4+}$ & $1.73374 \mathrm{e}-01$ & $8.92716 \mathrm{e}-07$ & $1.31408 \mathrm{e}-01$ & $8.41767 \mathrm{e}+03$ & 0.7375 & 2011.8704 \\
\hline $\mathrm{Th}^{4+}$ & $1.86519 \mathrm{e}-01$ & $3.23590 \mathrm{e}-06$ & $1.60896 \mathrm{e}-01$ & $2.68777 \mathrm{e}+03$ & 0.9030 & 642.3918 \\
\hline
\end{tabular}



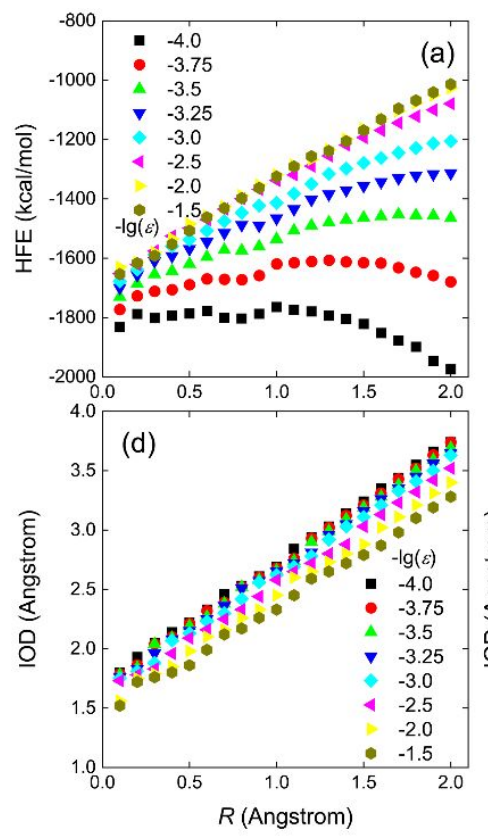
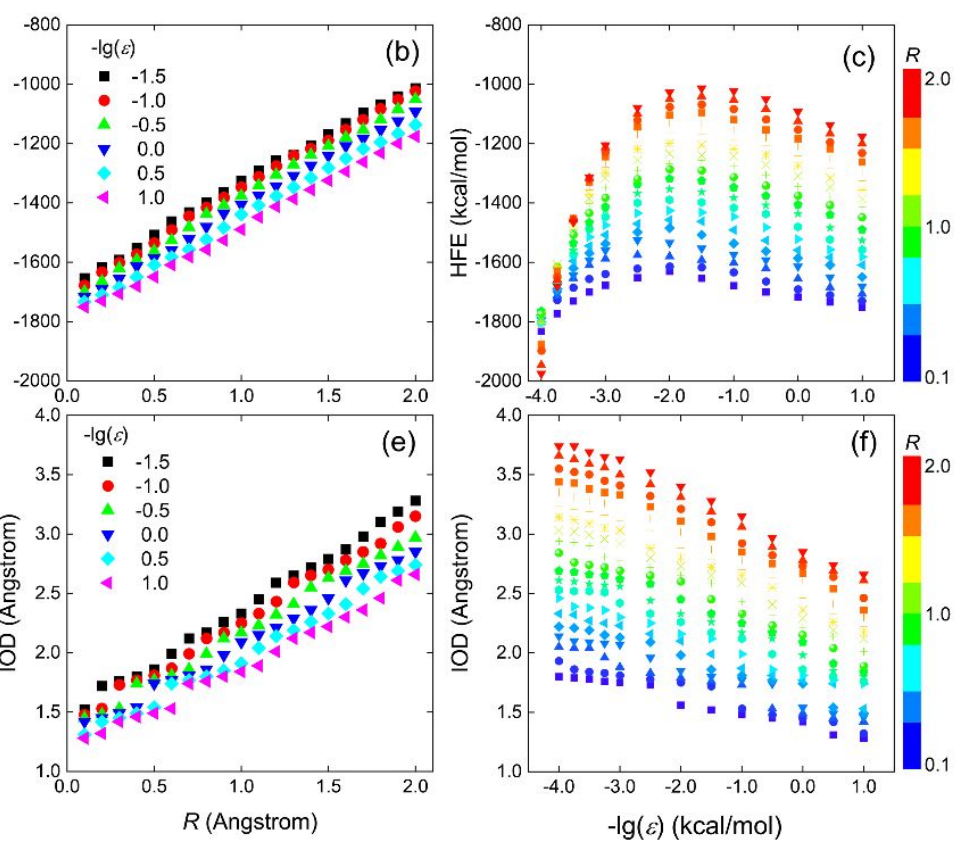

Figure S1. Hydration free energy (HFE) of the test M(IV) cation in TIP3P water model as a function of LJ scanning parameter $R$ for the scanning $-\lg (\varepsilon) \leq-1.5(\varepsilon \geq 31.6228$ $\mathrm{kcal} / \mathrm{mol}$, panel a) and $-\lg (\varepsilon) \geq-1.5$ (panel b) and of LJ parameter $-\lg (\varepsilon)$ (panel c). Ionoxygen distance (IOD) of the test ion as a function of $R$ and $-\lg (\varepsilon)$ is presented in panels d-f.
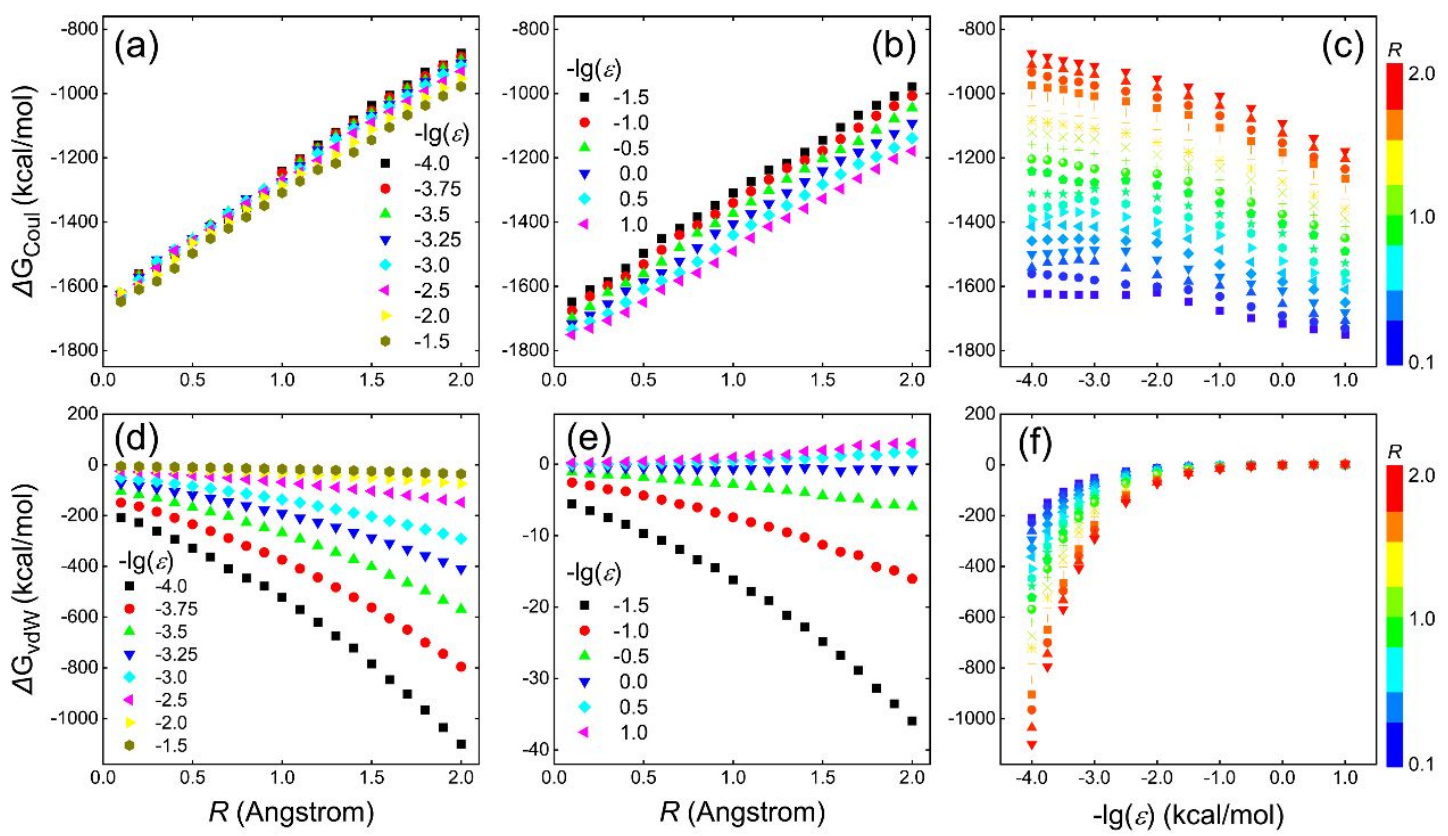

Figure S2. Coulomb ( $\Delta G_{\text {Coul }}$, a-c) and $\mathrm{vdW}\left(\Delta G_{\mathrm{vdW}}, \mathrm{d}-\mathrm{f}\right)$ contributions to hydration free energies (HFEs) of the test M(IV) cation in TIP3P water model as a function of LJ scanning parameters of $R$ and $\varepsilon$. 

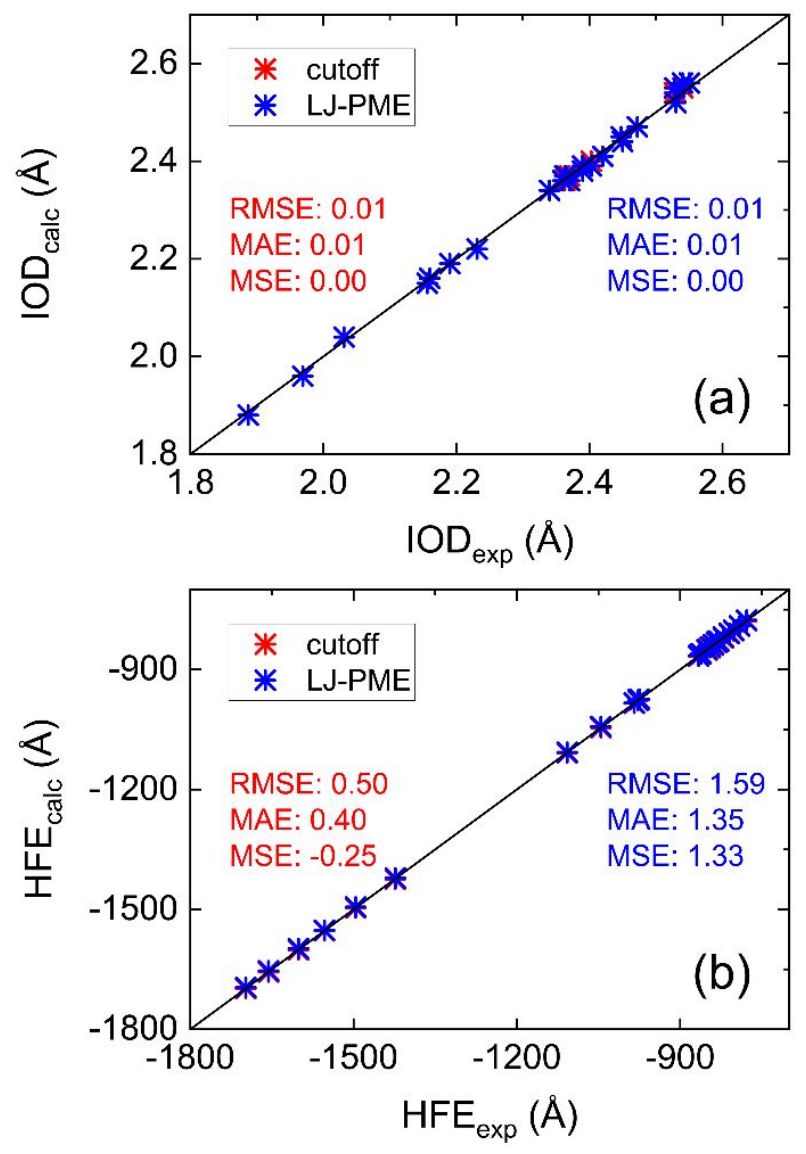

Figure S3. Comparison of calculated IOD (a) and HFE (b) of M(III) and M(IV) ions in TIP3P with experimental observations using two methods of cut-off and LJ-PME for vdW interactions.

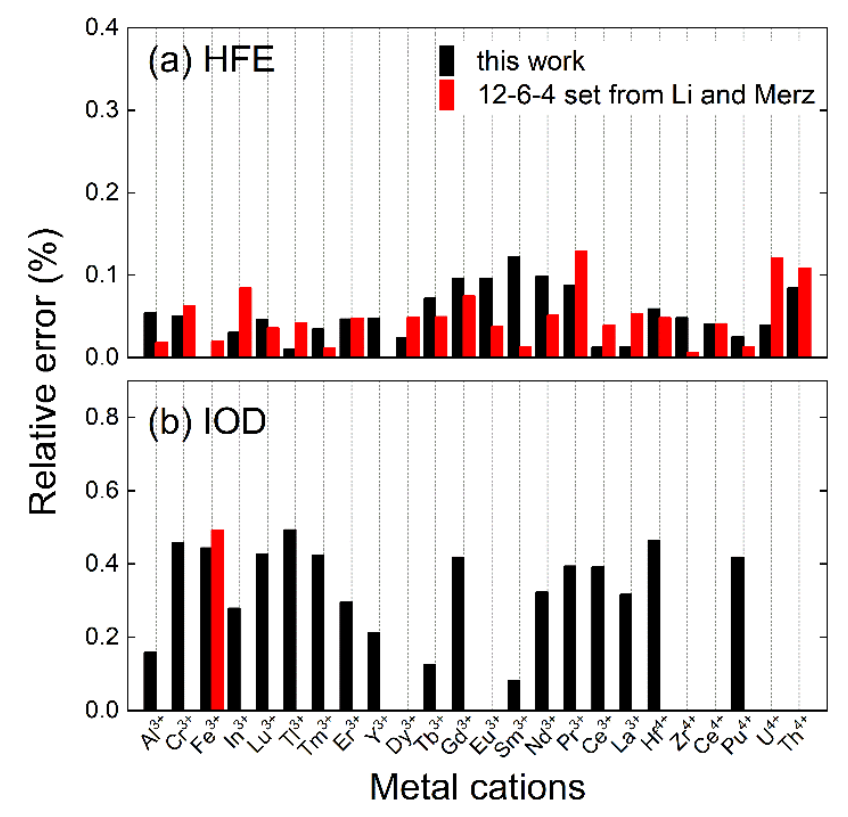

Figure S4. Relative errors of the calculated HFE (a) and IOD (b) values from experiment (Table 1) for the 24 metal cations in SPC/E water (Table 3) using the 12-6 LJ model in this work (black) and the 12-6-4 set by the group of Li and Merz. ${ }^{2}$ 


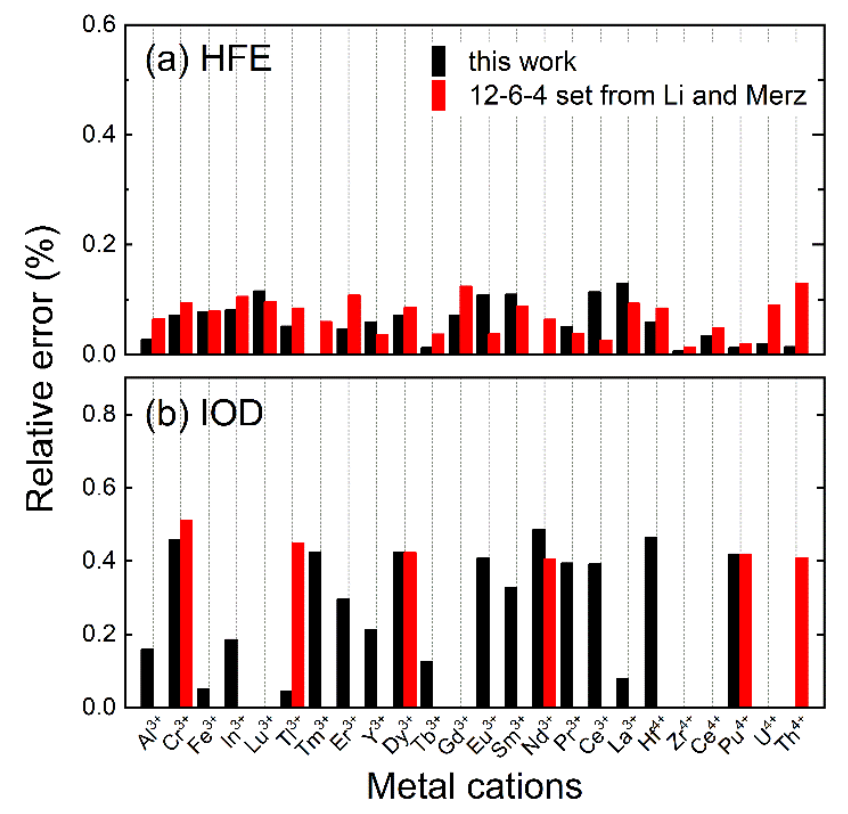

Figure S5. Relative errors of the calculated HFE (a) and IOD (b) values from experiment (Table 1) for the 24 metal cations in TIP4P-Ew water (Table 4) using the 12-6 LJ model in this work (black) and the 12-6-4 set by the group of Li and Merz. ${ }^{2}$

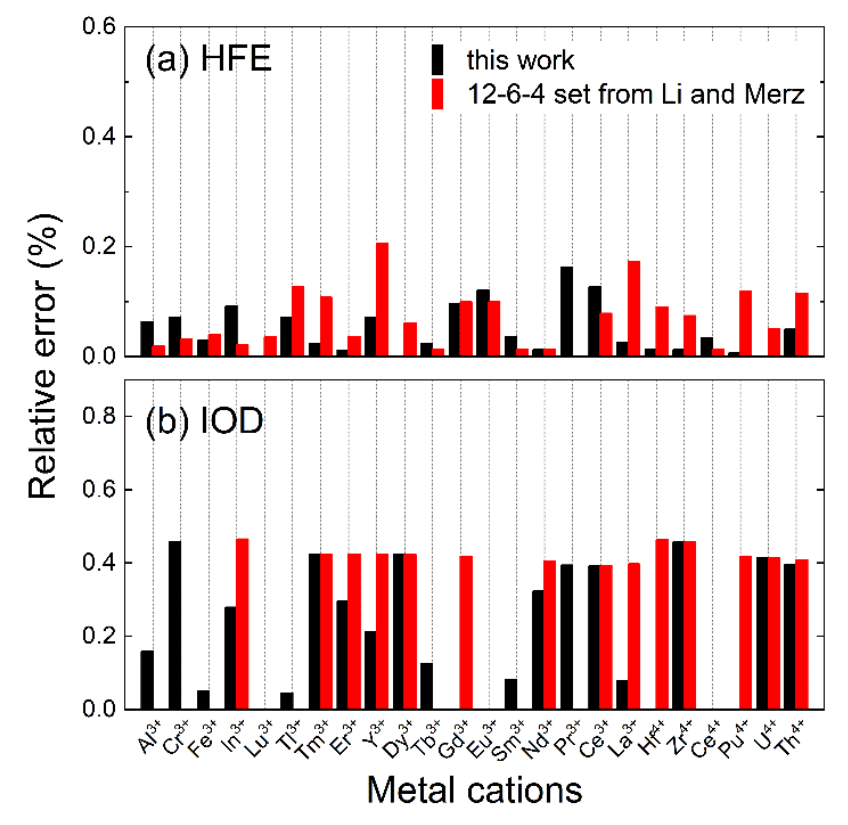

Figure S6. Relative errors of the calculated HFE (a) and IOD (b) values from experiment (Table 1) for the 24 metal cations in OPC3 water (Table 3) using the 12-6 LJ model in this work (black) and the 12-6-4 set by the group of Li and Merz. ${ }^{3}$ 


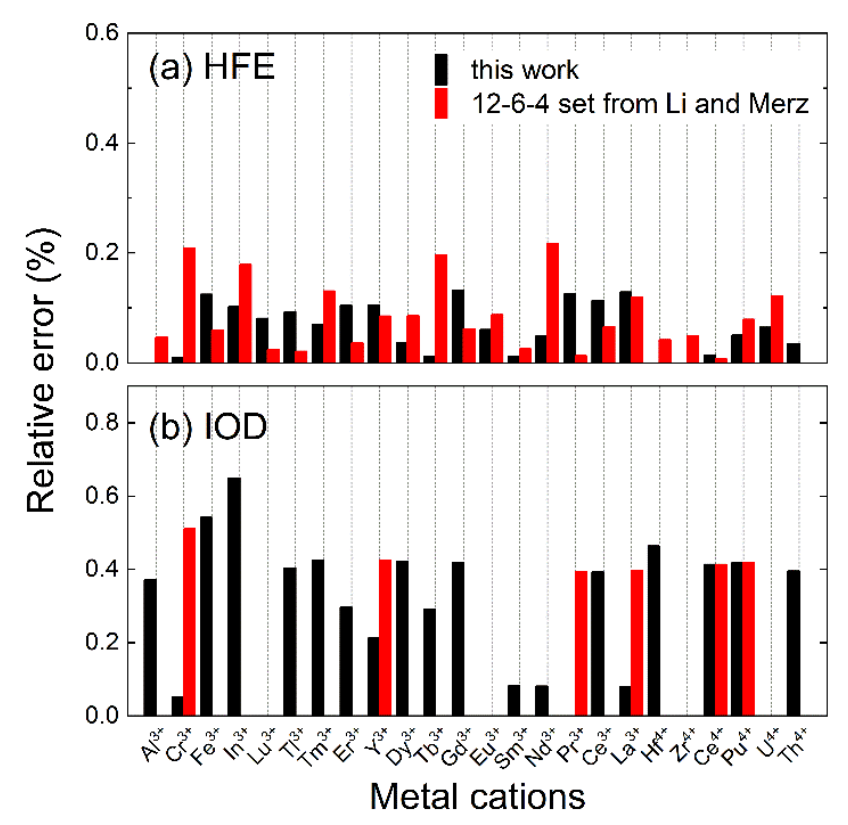

Figure S7. Relative errors of the calculated HFE (a) and IOD (b) values from experiment (Table 1) for the 24 metal cations in OPC water (Table 5) using the 12-6 LJ model in this work (black) and the 12-6-4 set by the group of Li and Merz. ${ }^{3}$

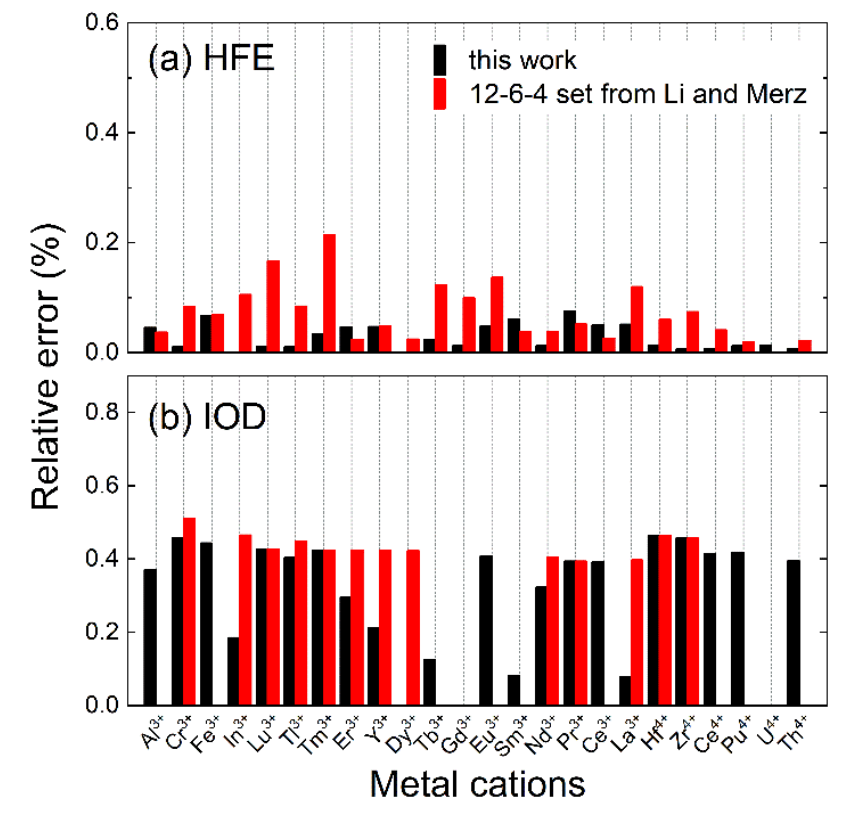

Figure S8. Relative errors of the calculated HFE (a) and IOD (b) values from experiment (Table 1) for the 24 metal cations in TIP3P-FB water (Table 3) using the 12-6 LJ model in this work (black) and the 12-6-4 set by the group of Li and Merz. ${ }^{3}$ 


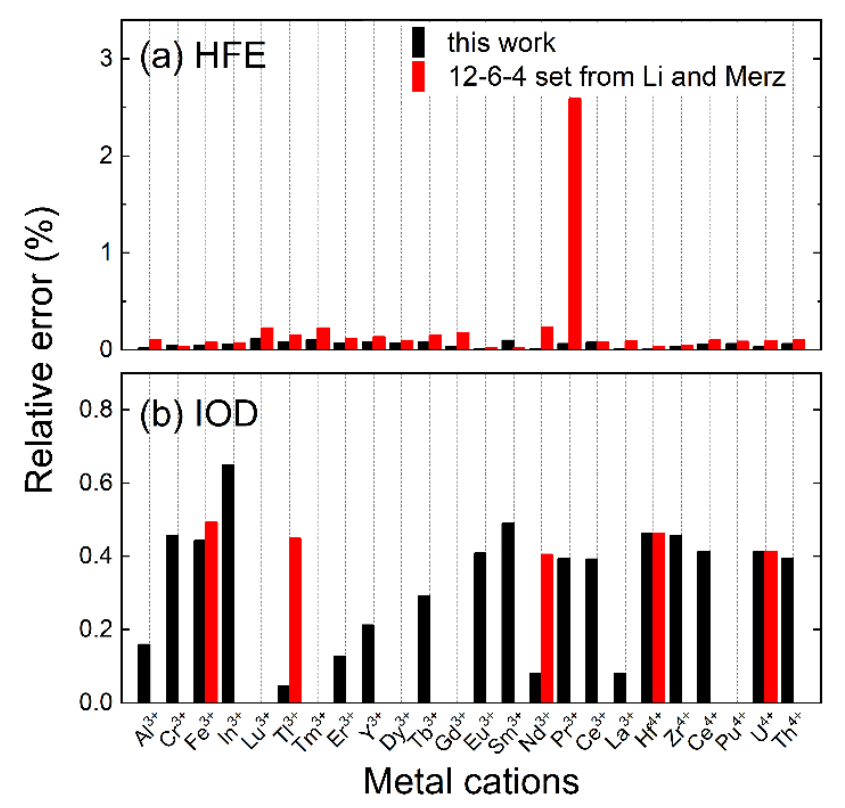

Figure S9. Relative errors of the calculated HFE (a) and IOD (b) values from experiment (Table 1) for the 24 metal cations in TIP4P-FB water (Table 5) using the 12-6 LJ model in this work (black) and the 12-6-4 set by the group of Li and Merz. ${ }^{3}$ 

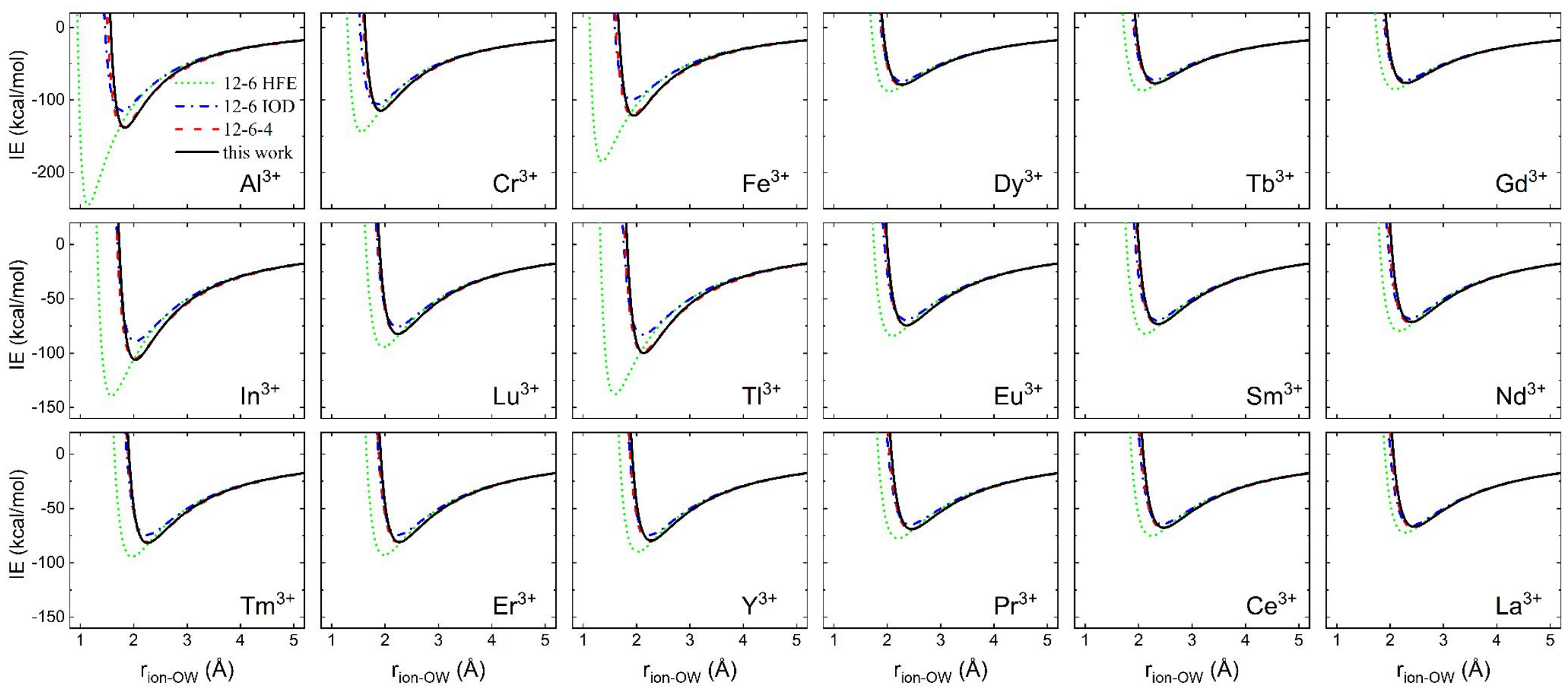

Figure S10. Interaction energy (IE) between M(III) ions and TIP3P water for different ion models from this work and the 12-6 HFE, 12-6 IOD, and 12-6-4 sets by the groups of Li and Merz. ${ }^{2}$ 

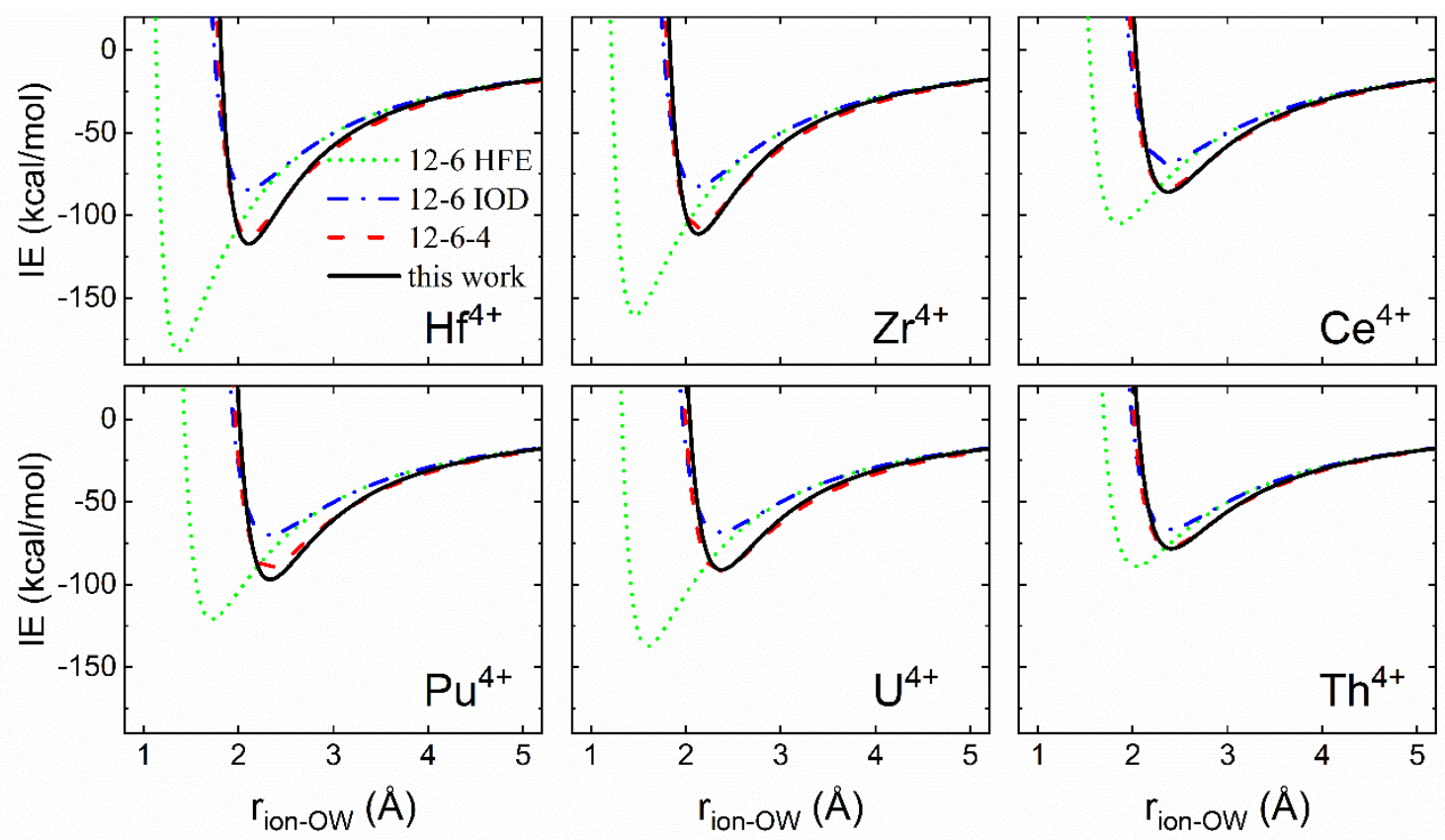

Figure S11. Interaction energy (IE) between M(IV) ions and TIP3P water for different ion models from this work and the 12-6 HFE, 12-6 IOD, and 12-6-4 sets by the groups of Li and Merz. ${ }^{2}$

\section{References}

(1) Shannon, R. Revised Effective Ionic Radii and Systematic Studies of Interatomic

Distances in Halides and Chalcogenides. Acta Crystallogr. A 1976, 32, 751-767.

(2) Li, P.; Song, L. F.; Merz, K. M. Parameterization of Highly Charged Metal Ions

Using the 12-6-4 LJ-Type Nonbonded Model in Explicit Water. J. Phys. Chem. B 2015, 119, 883-895.

(3) Li, Z.; Song, L. F.; Li, P.; Merz, K. M. Parametrization of Trivalent and

Tetravalent Metal Ions for the OPC3, OPC, TIP3P-FB, and TIP4P-FB Water Models.

J. Chem. Theory Comput. 2021, 17, 2342-2354. 
(4) Zeiss, G. D.; Meath, W. J. Dispersion Energy Constants C6(A, B), Dipole Oscillator Strength Sums and Refractivities for $\mathrm{Li}, \mathrm{N}, \mathrm{O}, \mathrm{H}_{2}, \mathrm{~N}_{2}, \mathrm{O}_{2}, \mathrm{NH}_{3}, \mathrm{H}_{2} \mathrm{O}, \mathrm{NO}$ and $\mathrm{N}_{2} \mathrm{O}$. Mol. Phys. 1977, 33, 1155-1176.

(5) Marcus, Y. Ionic Radii in Aqueous Solutions. Chem. Rev. 1988, 88, 1475-1498.

(6) Essmann, U.; Perera, L.; Berkowitz, M. L.; Darden, T.; Lee, H.; Pedersen, L. G. A Smooth Particle Mesh Ewald Method. J. Chem. Phys. 1995, 103, 8577-8593.

(7) Wennberg, C. L.; Murtola, T.; Hess, B.; Lindahl, E. Lennard-Jones Lattice Summation in Bilayer Simulations Has Critical Effects on Surface Tension and Lipid Properties. J. Chem. Theory Comput. 2013, 9, 3527-3537.

(8) Hess, B. Determining the Shear Viscosity of Model Liquids from Molecular Dynamics Simulations. J. Chem. Phys. 2002, 116, 209-217.

(9) Wensink, E. J. W.; Hoffmann, A. C.; van Maaren, P. J.; van der Spoel, D. Dynamic Properties of Water/Alcohol Mixtures Studied by Computer Simulation. $J$. Chem. Phys. 2003, 119, 7308-7317.

(10) Skinner, L. B.; Huang, C.; Schlesinger, D.; Pettersson, L. G. M.; Nilsson, A.; Benmore, C. J. Benchmark Oxygen-Oxygen Pair-Distribution Function of Ambient Water from X-Ray Diffraction Measurements with a Wide Q-Range. J. Chem. Phys. 2013, 138, 074506.

(11) Lide, D. R., Handbook of Chemistry and Physics, 90th; CRC: Boca Raton, FL: 2010. 
(12) Jorgensen, W. L.; Chandrasekhar, J.; Madura, J. D.; Impey, R. W.; Klein, M. L. Comparison of Simple Potential Functions for Simulating Liquid Water. J. Chem. Phys. 1983, 79, 926-935.

(13) Mills, R. Self-Diffusion in Normal and Heavy Water in the Range 1-45. Deg. J. Phys. Chem. 1973, 77, 685-688.

(14) Harris, K. R.; Woolf, L. A. Temperature and Volume Dependence of the Viscosity of Water and Heavy Water at Low Temperatures. J. Chem. Eng. Data 2004, 49, 1064-1069.

(15) Harris, K. R.; Woolf, L. A. Temperature and Volume Dependence of the Viscosity of Water and Heavy Water at Low Temperatures. J. Chem. Eng. Data 2004, $49,1851-1851$.

(16) Richter, U.; Brand, P.; Bohmhammel, K.; Könnecke, T. Thermodynamic Investigations of Aqueous Solutions of Aluminium Chloride. J. Chem. Thermodyn. $2000,32,145-154$.

(17) Mason, C. M. The Activity and Osmotic Coefficients of Trivalent Metal Chlorides in Aqueous Solution from Vapor Pressure Measurements at 25․ J. Am. Chem. Soc. 1938, 60, 1638-1647. 